\title{
Hamiltonian actions of unipotent groups on compact Kähler manifolds
}

\author{
Daniel Greb and Christian Miebach
}

\begin{abstract}
We study meromorphic actions of unipotent complex Lie groups on compact Kähler manifolds using moment map techniques. We introduce natural stability conditions and show that sets of semistable points are Zariski-open and admit geometric quotients that carry compactifiable Kähler structures obtained by symplectic reduction. The relation of our complex-analytic theory to the work of Doran-Kirwan regarding the Geometric Invariant Theory of unipotent group actions on projective varieties is discussed in detail.
\end{abstract}

Keywords. Unipotent algebraic groups; automorphisms of compact Kähler manifolds; Kähler metrics on fibre bundles and homogeneous spaces; moment maps; symplectic reduction; Geometric Invariant Theory

2010 Mathematics Subject Classification. 32M05; 32M10; 32Q15; 14L24; 14L30; 37J15; 53D20

[Français]

Titre. Actions hamiltoniennes des groupes unipotents sur les variétés kählériennes compactes

Résumé. Nous étudions les actions méromorphes de groupes de Lie complexes unipotents sur les variétés kählériennes compactes en utilisant des techniques de type application moment. Nous introduisons des conditions de stabilité naturelles et nous montrons que l'ensemble des points semi-stables forme un ouvert de Zariski et admet des quotients géométriques munis de structures kählériennes compactifiables obtenues par réduction symplectique. Le parallèle entre notre théorie analytique complexe et les travaux de Doran-Kirwan concernant la Théorie Géométrique des Invariants des actions de groupes unipotents sur les variétés projectives est discuté en détails.

Received by the Editors on May 8, 2018, and in final form on September 6, 2018.

Accepted on October 6, 2018.

Daniel Greb

Essener Seminar für Algebraische Geometrie und Arithmetik, Fakultät für Mathematik, Universität Duisburg-Essen, 45117 Essen, Germany

e-mail: daniel.greb@uni-due.de

Christian Miebach

Université du Littoral Côte d'Opale, EA 2797 - LMPA - Laboratoire de mathématiques pures et appliquées Joseph Liouville, F62228 Calais, France

e-mail: christian.miebach@univ-littoral.fr 


\section{Contents}

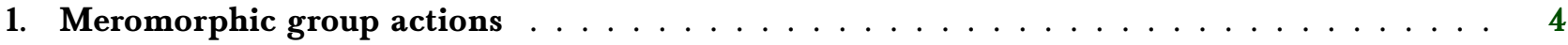

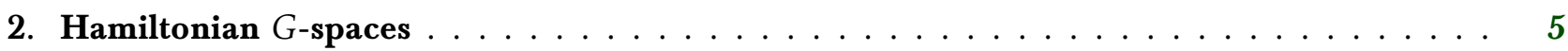

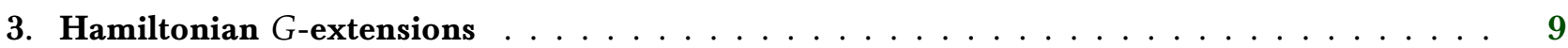

4. The set of semistable points with respect to unipotent groups $\ldots \ldots \ldots \ldots$

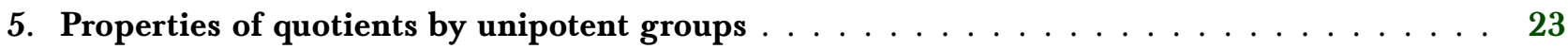

\section{Introduction}

Since the fundamental work of Mumford [MFK94], Kirwan [Kir84], Guillemin-Sternberg [GS82], and others, moment map geometry has become one of the most important tools for studying actions of complexreductive Lie groups $G=K^{\mathbb{C}}$ on Kähler manifolds. Given a Hamiltonian G-manifold, i.e., a Kähler Gmanifold $\left(X, \omega_{X}\right)$ admitting a moment map $\mu: X \rightarrow \operatorname{Lie}(K)^{*}$ for the $K$-action, by the work of HeinznerLoose [HL94], Heinzner-Huckleberry-Loose [HHL94], and Sjamaar [Sj95], the set of $\mu$-semistable points $X_{G}^{s S}(\mu):=\left\{x \in X \mid \overline{G \cdot x} \cap \mu^{-1}(0) \neq \emptyset\right\}$ admits an analytic Hilbert quotient, i.e., a G-invariant holomorphic Stein map $\pi: X_{G}^{s S}(\mu) \rightarrow X_{G}^{s S}(\mu) / / G$ onto a Kählerian complex space $X_{G}^{s S}(\mu) / / G$ with structure sheaf $\mathscr{O}_{X_{G}^{s s}(\mu) / / G}=\left(\pi_{*} \mathscr{O}_{X_{G}^{s s}(\mu)}\right)^{G}$; see also the survey [HH99]. If $X$ is projective algebraic, and if the Kähler form $\omega_{X}$ as well as the moment map $\mu$ are induced by an embedding of $X$ into some projective space, both the set $X_{G}^{s s}(\mu)$ of semistable points and the quotient $X_{G}^{s s}(\mu) / / G$ are the ones constructed via Geometric Invariant Theory (GIT). This theory crucially uses the fact that complex-analytic objects on $X$ can be averaged over the compact group $K$ to produce $G$-invariant objects, which then can be used to construct the quotient.

On the other hand, actions of unipotent groups on (compact) Kähler manifolds appear naturally in a number of contexts and play an important role in Kähler geometry. By a fundamental result of Lichnerowicz and Matsushima, a given compact Kähler manifold $X$ can admit a constant scalar curvature Kähler metric only if the Lie sub-algebra of all holomorphic vector fields having a zero is reductive, see e.g. [Sze14, Proposition 4.18 and Remark 4.12]. In other words, unipotent subgroups of $\operatorname{Aut}(X)$ appear as obstructions to the existence of such metrics. In a related direction, the paper [CD16] proposes a way to produce canonical destabilising test-configurations (showing $K$-unstability of $X$ ) from non-reductive subgroups of the automorphism group.

Motivated by these and other moduli-theoretic questions, Doran and Kirwan in [DK07] started to study actions of unipotent algebraic groups $N$ on projective manifolds $X$ linearised in very ample line bundles, using invariant-theoretic methods on the one hand and Geometric Invariant Theory for related actions of reductive groups $G$ containing $N$ on twisted products $G \times_{N} X$ on the other hand. When thinking about the relation of their work to Kähler geometry and moment maps, one encounters three basic questions:

(a) What is the correct analogue of a "linear" action in Kähler geometry?

(b) Given a compact Kähler $N$-manifold $X$, how can one produce Kähler metrics on the non-compact twisted product $G \times{ }_{N} X$ ?

(c) If (b) has a positive answer, can one use moment map geometry on the non-compact Hamiltonian $G$-manifold to produce quotients for the $N$-action on $X$ with good geometric and complex-analytic properties?

With a different set of problems in mind, Question (a) has been solved a rather long time ago by Fujiki [Fuj78], Lieberman [Lie78], and Sommese [Som75]: meromorphic actions and more generally actions for which the induced action on the Albanese torus is trivial were already called "linear" or "projective" 
in loc. cit., and it turns out that also with a view towards moment map geometry and Kählerian quotient theory, these are the correct conditions to impose, see Remark 2.2. For actions of reductive Lie groups this was observed by Huckleberry-Wurzbacher [HW90, Remark on page 262] and Fujiki [Fuj96, Lemma 2.1]. Note that the Lie algebra of the group of all automorphisms acting trivially on Albanese consists exactly of those holomorphic vector fields having a zero, e.g. see [Fuj78, Proposition 6.8], which connects the question of "linearity" to the one concerning the existence of extremal Kähler metrics discussed above.

\section{Main results}

As our first contribution, using a criterion of Blanchard and and properties of unipotent groups, with respect to Question (b) we prove the following result.

Theorem (Theorem 3.7). Let $N$ be a unipotent subgroup of the simply-connected complex semisimple Lie group $G$. For a connected compact Kähler manifold $\left(X, \omega_{X}\right)$ endowed with a holomorphic $N$-action the following statements are equivalent.

(1) There exists a Hamiltonian $G$-extension $\left(Z, \omega_{Z}\right)$ of the $N$-action on $X$.

(2) The $N$-action on $X$ is meromorphic.

(3) The twisted product $G \times_{N} X$ is Kähler.

Moreover, given a meromorphic $N$-action on $X$ we can always find a Hamiltonian $G$-extension that is a $G$ equivariant compactification of $G \times_{N} X$.

Here, a Hamiltonian G-extension of the $N$-action on $\left(X, \omega_{X}\right)$ consists of a connected compact Hamiltonian $G$-manifold $\left(Z, \omega_{Z}\right)$ and an $N$-equivariant embedding $\iota: X \hookrightarrow Z$ such that for the de Rham cohomology classes associated with the Kähler forms we have $\iota^{*}\left[\omega_{Z}\right]=\left[\omega_{X}\right]$. This theorem links the condition "meromorphic" to moment map geometry of the $G$-action on $G \times_{N} X$, and therefore opens the door to using the complex-reductive theory for the construction of quotients of $X$ with respect to the $N$-action.

Once the existence of Hamiltonian $G$-extensions is established, these can be used to define the set of $N$-semistable points $X_{N}^{s s}\left[\omega_{X}\right]$ with respect to the given Kähler class $\left[\omega_{X}\right] \in H^{2}(X, \mathbb{R})$, after one has chosen a suitable Kähler form on the quasi-affine homogeneous space $G / N$. While we show in Theorem 4.4, using Hodge-theoretic arguments as well as the relation of $G$-semistability to $K$-invariant strictly plurisubharmonic exhaustion functions, that this definition does not depend on the chosen $G$-extension, the choice of metric on $G / N$ influences semistability in a subtle way, as we explore in great detail in Section 4.C. The problems that occur are closely related to the ones encountered in the algebraic situation when searching for various kinds of "reductive envelopes", cf. [DK07, Sections 5.2 and 5.3], and can be traced back to the fact that in general there is no choice of metric on $G / N$ so that the corresponding moment map is proper or admissible in the sense of [Sj95]. A detailed comparison of the moment map approach introduced here and the GIT approach of Doran-Kirwan is presented in Section 4.E.

Finally, regarding Question (c), we establish that the set of $N$-semistable points indeed has a number of very desirable complex-geometric properties. The following result summarises the content of Theorem 5.3, Proposition 5.4, Theorem 5.5, and Theorem 5.6.

Theorem. Let $\left(X, \omega_{X}\right)$ be a compact Kähler manifold endowed with a meromorphic $N$-action. Then, the following holds.

(a) The set $X_{N}^{s s}\left[\omega_{X}\right]$ of semistable points admits a geometric quotient $\pi: X_{N}^{s s}\left[\omega_{X}\right] \rightarrow X_{N}^{s s}\left[\omega_{X}\right] / N$ by the $N$ action. In fact, $\pi$ is a principal $N$-fibre bundle and $X_{N}^{s S}\left[\omega_{X}\right] / N=: Q$ is smooth.

(b) The definition of semistability naturally induces an open embedding $\phi: Q=X_{N}^{s S}\left[\omega_{X}\right] / N \hookrightarrow \bar{Q}$ of $Q$ into a compact complex space $\bar{Q}$ such that $\bar{Q} \backslash \phi(Q)$ is analytic. 
(c) The set $X_{N}^{s s}\left[\omega_{X}\right]$ of semistable points is Zariski-open in $X$. Moreover, the quotient map $\pi: X_{N}^{s S}\left[\omega_{X}\right] \rightarrow Q$ extends to a meromorphic map $\pi: X \rightarrow \bar{Q}$.

(d) There exists a Kähler structure $\omega_{\bar{Q}}$ on $\bar{Q}$ whose restriction $\omega_{Q}=\left.\omega_{\bar{Q}}\right|_{Q}$ to $Q \hookrightarrow \bar{Q}$ is smooth and fulfils

$$
\left[\pi^{*} \omega_{Q}\right]=\left[\left.\omega_{X}\right|_{X_{N}^{s s}\left[\omega_{X}\right]}\right] \in H^{2}\left(X_{N}^{s s}\left[\omega_{X}\right], \mathbb{R}\right) .
$$

\section{Future directions}

With the fundamental results of a Kählerian quotient theory for meromorphic actions of unipotent groups established, interesting questions include whether despite the difficulties presented by the examples collected in Section 4.3 under certain additional conditions the set of semistable points can be shown to be independent of further choices, whether in certain applications there are natural Kähler metrics on the homogeneous space $G / N$ leading to a quotient that is as well-adapted as possible to the given geometric situation at hand, and whether given a compact Kähler manifold $\left(X, \omega_{X}\right)$ with non-trivial non-reductive part in the automorphism group (obstructing the existence of special metrics) one can use the Kählerian quotient theory for this unipotent group in order to produce a Kählerian complex space/manifold where said obstruction vanishes and special metrics might exist.

\section{Organisation of the paper}

In the first two sections we review basic facts about meromorphic actions and GIT on Kähler manifolds via moment maps. In Section 3 we prove our first main result, Theorem 3.7. The difficulties one encounters in defining the set of semistable points for holomorphic actions of unipotent groups, the actual definition of semistability, as well as fundamental properties of the set of semistable points are discussed in Section 4, while in the final Section 5 we prove the second main result concerning the properties of semistable quotients for unipotent group actions.

\section{Acknowledgements}

The first author wants to thank the Laboratoire de Mathématiques Pures et Appliquées at Université du Littoral Côte d'Opale as well as FRIAS for hospitality during research visits in the spring of 2016 and 2018, respectively. During the preparation of this paper, he has been partially supported by the DFGCollaborative Research Center SFB/TR 45 "Periods, moduli spaces and arithmetic of algebraic varieties". The second author gratefully acknowledges the hospitality of the mathematical departments of the universities of Duisburg-Essen and Freiburg as well as of the FRIAS during the spring of 2016, 2017 and 2018. Both authors would like to thank Peter Heinzner for fruitful and interesting discussions.

\section{Global conventions}

We work over the field $\mathbb{C}$ of complex numbers. A complex space is a reduced complex space with countable topology. Analytic subsets are assumed to be closed. Manifolds are assumed to be connected.

\section{Meromorphic group actions}

Let us review some facts about meromorphic group actions from [Fuj78] that we will use freely in the following. Lieberman obtained essentially the same results in [Lie78]. In this section, $G$ denotes a complex Lie group.

A meromorphic structure on the complex Lie group $G$ is a compactification $\bar{G}$ together with a meromorphic mapping $\bar{\mu}: \bar{G} \times \bar{G} \rightarrow \bar{G}$ which extends the group multiplication of $G$ such that $\bar{\mu}$ is holomorphic on $(G \times \bar{G}) \cup(\bar{G} \times G)$. In other words, the compactification $\bar{G}$ is $(G \times G)$-equivariant. Moreover, the map $G \rightarrow G$, $g \mapsto g^{-1}$, extends to a meromorphic map $\bar{G} \rightarrow \bar{G}$. 
Let us fix a meromorphic structure on $G$. A complex subgroup $H$ of $G$ is meromorphic if the topological closure $\bar{H}$ of $H$ in $\bar{G}$ is analytic.

Remark 1.1. If $G$ is linear algebraic, we may and will choose $\bar{G}$ to be a projective manifold. The meromorphic subgroups of $G$ are then precisely the algebraic subgroups of $G$.

Let $X$ be a complex space endowed with a holomorphic $G$-action. This $G$-action is meromorphic if the action map $G \times X \rightarrow X$ extends to a meromorphic map $\bar{G} \times X \rightarrow X$. For meromorphic actions on compact Kähler manifolds (in fact, on reduced compact complex spaces of class $\mathcal{C}$ ) we have the following quotient theorem, see [Fuj78, Theorem 4.1].

Theorem 1.2. (Quotient Theorem) Let $X$ be a compact Kähler manifold on which $G$ acts meromorphically. Then, there exist a compact complex space $Y$ and a G-invariant surjective meromorphic map $\pi: X \rightarrow Y$ such that the following universal property is satisfied. If $\pi^{\prime}: X \rightarrow Y^{\prime}$ is another $G$-invariant meromorphic map to a compact complex space $Y^{\prime}$, then there exists a unique meromorphic map $m: Y \rightarrow Y^{\prime}$ such that $m \circ \pi=\pi^{\prime}$. In this situation we call $\pi: X \rightarrow Y$ a meromorphic quotient for the G-action on $X$.

Example 1.3. Let $H$ be an algebraic subgroup of $G$. Then there exists a $G$-equivariant smooth projective compactification $\overline{G / H}$ of $G / H$. Moreover, the $H$-principal bundle $\pi: G \rightarrow G / H$ extends to a rational map $\bar{\pi}: \bar{G} \rightarrow \overline{G / H}$. Then $\bar{\pi}$ is a meromorphic quotient for the $H$-action on $\bar{G}$.

In fact, by inspecting Fujiki's construction, one obtains the following more precise result that has appeared in several places in the literature; see for example [BBS85, Theorem 0.2.2], as well as [Grel0b, Proposition 3.1], [Hu05, Section 3], and the references given there for the analogous result in the algebraic category.

Proposition 1.4. Let $X$ be a compact Kähler manifold on which $G$ acts meromorphically. Then, there exists an irreducible, compact analytic subset $Q_{F}$ of the cycle space of $X$, a G-invariant meromorphic map $\pi_{F}: X \rightarrow Q_{F}$, and a $G$-invariant Zariski-open subset $U_{F} \subset \operatorname{dom}\left(\pi_{F}\right)$, called a Fujiki set of $X$, such that

(1) $\pi_{F}: X \rightarrow Q_{F}$ is a meromorphic quotient for the $G$-action on $X$,

(2) $U_{F} \subset X_{\text {gen }}:=\{x \in X \mid \operatorname{dim} G \cdot x=m$ is maximal $\}$,

(3) for all $u \in U_{F}$, we have $\pi_{F}(u)=\overline{G \cdot u}$, considered as a (reduced) cycle of $X$,

(4) $\pi_{F}\left(U_{F}\right)$ is smooth and Zariski-open in $Y$, and the restriction $\left.\pi_{F}\right|_{U_{F}}: U_{F} \rightarrow \pi_{F}\left(U_{F}\right)$ is a geometric quotient.

We call $\pi_{F}: X \rightarrow Q_{F}$ a Fujiki quotient of $X$ by $G$.

\section{Hamiltonian G-spaces}

Let $G=K^{\mathbb{C}}$ be a complex reductive group with maximal compact subgroup $K$. In this section we review the definition and some properties of Hamiltonian $G$-manifolds. A general reference for the complex-analytic theory of moment maps on Kähler manifolds is [HH99].

\section{A. Moment maps}

Let $\left(Z, \omega_{Z}\right)$ be a Kähler manifold with Kähler form $\omega_{Z}$. Suppose that $G$ acts holomorphically on $Z$ such that $\omega_{Z}$ is $K$-invariant. A moment map for the $K$-action on $Z$ is a $K$-equivariant smooth map $\mu: Z \rightarrow k^{*}$, where $K$ acts via the coadjoint representation on the dual $k^{*}$ of its Lie algebra, such that

$$
d \mu^{\xi}=\iota_{\xi_{Z}} \omega_{Z}
$$


Here, for every $\xi \in \mathfrak{k}$, we write $\mu^{\xi} \in \mathcal{C}^{\infty}(Z)$ for the function defined by $\mu^{\xi}(z)=\mu(z)(\xi)$, and $\xi_{Z}$ for the vector field on $Z$ whose flow is given by $(t, z) \mapsto \exp (-t \xi) \cdot z$, and $\iota_{\xi_{Z}} \omega_{Z}$ for the contraction of $\omega_{Z}$ by $\xi_{Z}$.

If a moment map for the $K$-action on $Z$ exists, we say that $\left(Z, \omega_{Z}\right)$ is a Hamiltonian G-manifold. The notions introduced above make sense for actions of $G$ on Kählerian complex spaces, see for example [Gre10a, Sections 3.1 and 3.2] and the references given there; in this setup one speaks about Hamiltonian G-spaces.

Remark 2.1. If $G$ is semisimple, then every Kähler manifold $\left(Z, \omega_{Z}\right)$ endowed with a holomorphic $G$-action such that $\omega_{Z}$ is $K$-invariant is Hamiltonian. Moreover, in this case the moment map $\mu: Z \rightarrow k^{*}$ is unique, see [GS84, Proposition 24.1] and the discussion following this proposition.

Remark 2.2. For a compact Kähler manifold $\left(Z, \omega_{Z}\right)$ endowed with a holomorphic action of the connected complex reductive Lie group $G=K^{\mathbb{C}}$ and $K$-invariant Kähler form $\omega_{Z}$ the following statements are equivalent:

(1) $Z$ is a Hamiltonian G-manifold.

(2) $G$ acts meromorphically on $Z$ in the sense of [Fuj78], see also Section 1.

(3) $G$ acts trivially on the Albanese torus $\operatorname{Alb}(Z)$ of $Z$.

The equivalence $(1) \Longleftrightarrow(3)$ was observed in [Fuj96, Lemma 2.1 and subsequent remark]. The implication (2) $\Longrightarrow$ (3) follows from [Fuj78, Lemma 3.8] since every complex reductive group is linear algebraic. The last implication (3) $\Longrightarrow$ (2) follows from [Som75, Proposition I], see also [Fuj78, Proposition 6.10].

For later use we record an elementary result on the moment image of a Hamiltonian $G$-manifold $\left(Z, \omega_{Z}\right)$ with moment map $\mu: Z \rightarrow \mathfrak{k}^{*}:$ If the interior of $\mu(Z)$ relative to $\mathfrak{k}^{*}$ is non-empty, then, due to Sard's Theorem, there exists a point $z \in Z$ such that $d \mu_{z}$ is surjective. Since condition (2.1) implies

$$
\operatorname{ker} d \mu_{z}=(\mathfrak{k} \cdot z)^{\perp_{\omega_{Z}}}=\left\{\xi_{Z}(z) \mid \xi \in \mathfrak{k}\right\}^{\perp_{\omega_{Z}}},
$$

cf. [HH99, Section 2.3], and therefore that the $\operatorname{rank}$ of $\mu$ in $z$ coincides with $\operatorname{dim} K \cdot z$, we conclude that $K_{z}$ is finite. Conversely, if $K_{z}$ is finite, then $\mu$ is a submersion in $z$, hence the image of $\mu$ has interior points in $\mathfrak{k}^{*}$. We summarize this discussion in the following

Lemma 2.3. Suppose that $\left(Z, \omega_{Z}\right)$ is a G-connected ${ }^{1}$ Hamiltonian G-manifold. Then $\mu(Z)$ has non-empty interior in $\mathrm{k}^{*}$ if and only if $K$ acts with generically finite isotropy on $Z$.

\section{B. The set of semistable points}

The set of semistable points is defined by

$$
Z_{G}^{s S}(\mu):=\left\{z \in Z \mid \overline{G \cdot z} \cap \mu^{-1}(0) \neq \emptyset\right\} .
$$

The $G$-invariant set $Z_{G}^{s S}(\mu)$ is open and can be characterized in the following way. For $z \in Z_{G}^{s s}(\mu)$ consider the inclusion $\iota: \overline{G \cdot z} \cap Z_{G}^{s S}(\mu) \hookrightarrow Z$. Then $\iota^{*} \omega_{Z}=i \partial \bar{\partial} \rho$ for some strictly plurisubharmonic exhaustion function $\rho$ and $\iota^{*} \mu=\mu_{\rho}$ where $\mu_{\rho}: \overline{G \cdot z} \cap Z_{G}^{s s}(\mu) \rightarrow \mathfrak{k}^{*}$ is given by

$$
\mu_{\rho}(z)(\xi):=d \rho_{z}\left(J \xi_{Z}(z)\right)
$$

where $J$ denotes the complex structure of $Z$, see [HH96, Section 3].

If $Z$ is compact and if $G$ is semisimple, then $Z_{G}^{s s}(\mu)$ depends only on the Kähler class $\left[\omega_{Z}\right]$, see [HH96, p. 71]. Since we generalize this result later on for Hamiltonian actions of unipotent groups, we repeat its proof here for the readers' convenience.

\footnotetext{
$1 \uparrow$ We say that $Z$ is $G$-connected if it cannot be written as the disjoint union of two non-empty $G$-stable closed subsets.
} 
Proposition 2.4. Let $\left(Z, \omega_{Z}\right)$ be a compact Hamiltonian $G$-manifold with moment map $\mu$ and let $\omega_{Z}^{\prime}$ be another $K$-invariant Kähler form on $Z$ such that $\left[\omega_{Z}\right]=\left[\omega_{Z}^{\prime}\right] \in H^{2}(Z, \mathbb{R})$. Then there exists a moment map $\mu^{\prime}$ for the $K$-action on $\left(Z, \omega_{Z}^{\prime}\right)$ such that $Z_{G}^{s S}(\mu)=Z_{G}^{s S}\left(\mu^{\prime}\right)$.

Proof. Since $Z$ is compact Kähler, the $\partial \bar{\partial}$-lemma implies $\omega_{Z}^{\prime}=\omega_{Z}+i \partial \bar{\partial} \varphi$ for some $\varphi \in \mathcal{C}^{\infty}(Z)$. Defining $\mu_{\varphi}: Z \rightarrow \mathfrak{k}^{*}$ in the same way as in Equation (2.3) one checks directly that the map $\mu^{\prime}:=\mu+\mu_{\varphi}$ is a moment map for the $K$-action on $\left(Z, \omega_{Z}^{\prime}\right)$.

Now suppose that $z \in Z_{G}^{s s}\left(\omega_{Z}\right)$. As noted above, we have $\iota^{*} \omega_{Z}=i \partial \bar{\partial} \rho$ and $\iota^{*} \mu=\mu_{\rho}$ for some strictly plurisubharmonic function $\rho$ on $\overline{G \cdot z} \cap Z_{G}^{s S}(\mu)$ where $\iota: \overline{G \cdot z} \cap Z_{G}^{s S}(\mu) \hookrightarrow Z$ is the inclusion. Since $Z$ is assumed to be compact, the function $\varphi$ is bounded, hence $\rho+l^{*} \varphi$ is still an exhaustion function on $\overline{G \cdot z} \cap Z_{G}^{s S}(\mu)$. Consequently, $\iota^{*} \mu^{\prime}=\mu_{\rho+l^{*} \varphi}$ has a zero on $\overline{G \cdot z} \cap Z_{G}^{s S}(\mu)$, which implies $z \in Z_{G}^{s S}\left(\mu^{\prime}\right)$. The converse inclusion follows by symmetry.

Remark 2.5. If $G$ is semisimple and if $\left(Z, \omega_{Z}\right)$ is a compact Hamiltonian $G$-manifold, the moment map for the $K$-action on $Z$ is unique, see Remark 2.1 above. Due to Proposition 2.4, the set of semistable points therefore depends only on $\left[\omega_{Z}\right] \in H^{2}(Z, \mathbb{R})$. In this case we thus write $Z_{G}^{s s}\left[\omega_{Z}\right]$ instead of $Z_{G}^{s s}(\mu)$.

\section{C. Analytic Hilbert quotients}

The importance of semistability stems from the fact that the set of semistable points admits the analogue of a good quotient in the analytic category:

Let $G$ be a complex reductive Lie group and $Z$ a complex space endowed with a holomorphic $G$-action. A complex space $Y$ together with a $G$-invariant surjective holomorphic map $\pi: Z \rightarrow Y$ is called an analytic Hilbert quotient ${ }^{2}$ of $Z$ by the action of $G$ if

(1) $\pi$ is a locally Stein map, and

(2) $\left(\pi_{*} \mathscr{O}_{Z}\right)^{G}=\mathscr{O}_{Y}$ holds.

Here, locally Stein means that there exists an open covering of $Y$ by open Stein subspaces $U_{\alpha}$ such that $\pi^{-1}\left(U_{\alpha}\right)$ is a Stein subspace of $Z$ for all $\alpha$; by $\left(\pi_{*} \mathscr{O}_{Z}\right)^{G}$ we denote the sheaf $U \mapsto \mathscr{O}_{Z}\left(\pi^{-1}(U)\right)^{G}=\{f \in$ $\mathscr{O}_{Z}\left(\pi^{-1}(U)\right) \mid f$ G-invariant $\}, U$ open in $Y$.

An analytic Hilbert quotient of a holomorphic $G$-space $Z$ is unique up to biholomorphism once it exists and we will denote it by $Z / / G$. The following properties follow from the corresponding ones in the Stein case, where analytic Hilbert quotients always exist, see [Hei91]: Two points $x, x^{\prime} \in Z$ have the same image in $Z / / G$ if and only if $\overline{G \cdot x} \cap \overline{G \cdot x^{\prime}} \neq \emptyset$. For each $q \in Z / / G$, the fibre $\pi^{-1}(q)$ contains a unique closed $G$-orbit $G \cdot x$. The stabiliser $G_{x}$ of $x$ in $G$ is a complex reductive Lie group, see [Mat60]. If $A \subset X$ is a $G$-invariant analytic subset, then $\pi(A) \subset X / / G$ is analytic, and $\left.\pi\right|_{A}: A \rightarrow \pi(A)$ is an analytic Hilbert quotient.

The main results in the quotient theory for complex reductive group actions on Kähler spaces are summarised in the following theorem.

Theorem 2.6. ([HL94], [HHL94], [HH99], [Sj95]) Let $Z$ be a Hamiltonian G-space with Kähler form $\omega_{Z}$ and moment map $\mu: Z \rightarrow \mathfrak{k}^{*}$. Then,

(1) $Z_{G}^{s S}(\mu)$ is open and $G$-invariant, and the analytic Hilbert quotient $\pi: Z_{G}^{s s}(\mu) \rightarrow Z_{G}^{s s}(\mu) / / G$ exists,

(2) the inclusion $\mu^{-1}(0) \hookrightarrow Z_{G}^{s S}(\mu)$ induces a homeomorphism $\mu^{-1}(0) / K \simeq Z_{G}^{s S}(\mu) / / G$,

(3) the complex space $Z_{G}^{s s}(\mu) / / G$ carries a Kähler structure that is induced by symplectic reduction from $\omega_{Z}$ and that is smooth along a natural stratification of $Z_{G}^{s s}(\mu) / / G$.

$2 \uparrow$ In some places in the literature, the terminology semistable quotient is used for the same concept. 


\section{D. Moment maps associated with representations and their images}

Let $G$ be a connected semisimple complex Lie group acting linearly on a finite dimensional complex vector space $V$. If we equip $V$ with the $K$-invariant flat Kähler metric $\omega_{V}$ given by a $K$-invariant hermitian inner product $\langle\cdot, \cdot\rangle$, then a moment map $\mu_{V}: V \rightarrow \mathfrak{k}^{*}$ for the $K$-action on $V$ is given by $\mu_{V}^{\xi}(v)=-\frac{i}{2}\langle\xi . v, v\rangle$ for every $\xi \in \mathfrak{k}$. Note that $\omega_{V}=i \partial \bar{\partial} \rho$ where $\rho(v)=\|v\|^{2}$ for all $x \in V$ and that $\mu_{V}=\mu_{\rho}$ in this case.

For any $v \in V$ consider the affine $G$-variety $\overline{G \cdot v}$. The restriction of $\mu_{V}$ to $\overline{G \bullet v}$ yields the moment map for the $K$-action on $\overline{G \bullet v}$ associated with the strictly plurisubharmonic exhaustion function $\left.\rho\right|_{G \cdot v}$. By abuse of notation we will denote the restricted Kähler form and moment map again by $\omega_{V}$ and $\mu_{V}$, respectively. For later use we record the following result of Sjamaar, see [Sj98, Theorem 4.9, Lemma 4.10]. For its statement we have to introduce a maximal torus $T$ of $K$ with Lie algebra $t$, the choice of a positive Weyl chamber $\mathrm{t}_{+}^{*}$, and the corresponding set $\Lambda^{+}$of dominant weights. For $\lambda \in \Lambda^{+}$let $V_{\lambda}$ denote the irreducible $G$-representation with highest weight $\lambda$.

Theorem 2.7. The moment map $\mu_{V}: \overline{G \cdot v} \rightarrow \mathfrak{k}^{*}$ is proper and verifies

$$
\mu_{V}(\overline{G \cdot v}) \cap \mathrm{t}_{+}^{*}=\text { cone }\left\{\lambda \in \Lambda^{+} \mid V_{\lambda} \text { occurs in } \mathbb{C}[\overline{G \cdot v}]\right\} .
$$

In general it may be rather difficult to decide for which dominant weight $\lambda$ the irreducible representation $V_{\lambda}$ occurs in $\mathbb{C}[G \cdot v]$. Note that the inclusion $G \cdot v \hookrightarrow \overline{G \cdot v}$ gives an injective homomorphism of algebras $\mathbb{C}[\overline{G \cdot v}] \rightarrow \mathbb{C}[G \cdot v] \cong \mathbb{C}[G]^{G_{v}}$. Therefore the situation is slightly easier for affine completions of quasi-affine homogeneous spaces $G / G_{v}$ for which the map $\mathbb{C}[\overline{G \cdot v}] \rightarrow \mathbb{C}[G]^{G_{v}}$ is an isomorphism.

To follow this train of thought, recall that an algebraic subgroup $H$ of $G$ is called Grosshans if $G / H$ is quasi-affine and if the algebra $\mathbb{C}[G]^{H} \cong \mathbb{C}[G / H]$ is finitely generated. This is equivalent to the existence of a finite-dimensional $G$-representation space $W$ containing $G / H$ as an orbit $G \cdot w$ such that the codimension of $\overline{G \cdot w} \backslash G \cdot w$ in $\overline{G \cdot w}$ is at least 2, see [Gr97, Theorem 4.3]. Recall that an algebraic subgroup of $G$ is called unipotent if it consists entirely of unipotent elements. If $N$ is a unipotent subgroup of $G$, then $G / N$ is always quasi-affine, see [Gr97, Corollary 1.5], but not affine, see [Mat60]; however, not every such $N$ is Grosshans. If $N$ is the unipotent radical of a parabolic subgroup of $G$, then $N$ is Grosshans, see [Gr83, Theorem 2.2].

Suppose that the unipotent subgroup $N$ of $G$ is Grosshans. Then we have a canonical affine completion $\overline{G / N}^{\mathrm{a}}=\operatorname{Spec} \mathbb{C}[G]^{N}$. Since $N$ is contained in a maximal unipotent subgroup of $G$, we can deduce from [Sj98, Example 4.19] that

$$
\left\{\lambda \in \Lambda^{+} \mid V_{\lambda} \text { occurs in } \mathbb{C}[G]^{N}\right\}=\Lambda^{+} .
$$

Consequently, by embedding $\overline{G / N}^{\mathrm{a}}$ into any G-representation, we can find a Kähler form inducing a proper, surjective moment map for the $K$-action on $\overline{G / N}$. Combining this observation with an application of Lemma 2.3 to the free $K$-action on $G / N \subset \overline{G / N}^{\text {a }}$ we obtain the following result.

Lemma 2.8. Suppose that $N$ is a unipotent Grosshans subgroup of $G$. Then there exists a Kähler form $\omega_{V}$ on $G / N$ such that the image of the corresponding moment map $\mu_{V}: G / N \rightarrow k^{*}$ is a $K$-invariant dense open subset of $\mathfrak{k}_{\mathrm{reg}}^{*}$. Moreover, $\omega_{V}$ and $\mu_{V}$ extend to the canonical affine completion $\overline{\mathrm{G}} / \mathrm{N}^{a}$.

As the following example shows, this result depends on the choice of the $G$-representation into which we embed $G / N$.

Example 2.9. Let $G$ be a simply-connected semisimple complex Lie group. For any choice of $\lambda_{1}, \ldots, \lambda_{k} \in$ $\Lambda^{+}$consider $v:=v_{1}+\cdots+v_{k} \in V_{\lambda_{1}}^{*} \oplus \cdots \oplus V_{\lambda_{k}}^{*}$ where $v_{j} \in V_{\lambda_{j}}^{*}$ is a highest weight vector. Then we have $\mathbb{C}[\overline{G \cdot v}]=\bigoplus_{\lambda \in M} V_{\lambda}$ where $M$ is the submonoid of $\Lambda^{+}$generated by $\lambda_{1}, \ldots, \lambda_{k}$, see [PV72, Theorem 6]. Consequently, $\mu_{V}(\overline{G \cdot v}) \cap t^{*}$ is the cone generated by $\lambda_{1}, \ldots, \lambda_{k}$. 
To construct an example where the image of $\overline{G \cdot v}$ under the moment map is not the whole of $\mathfrak{k}^{*}$, let $G=\operatorname{SL}(3, \mathbb{C})$ and choose $\lambda_{1}=\omega_{1}+\omega_{2}$ and $\lambda_{2}=2 \omega_{1}+\omega_{2}$ where $\omega_{1}, \omega_{2}$ are the fundamental weights of $G$. Note that $V_{\lambda_{1}}$ and hence also $V_{\lambda_{1}}^{*}$ are isomorphic to the adjoint representation of $G=\operatorname{SL}(3, \mathbb{C})$. It follows that $G_{v_{1}}$ is the connected subgroup of $G$ having as Lie algebra the semi-direct sum of the kernel of $\lambda_{1}$ in the chosen Cartan sub-algebra of $\mathfrak{g}$ and the positive maximal unipotent sub-algebra $\mathfrak{n}$ of $\mathfrak{g}$. Since the Lie algebra of $G_{v_{2}}$ for $v_{2} \in V_{\lambda_{2}}$ contains $\mathfrak{n}$ and since the kernels of $\lambda_{1}$ and $\lambda_{2}$ intersect only trivially, the Lie algebra of the stabiliser of $v=v_{1}+v_{2}$ must coincide with $\mathfrak{n}$. In summary, we see that in the chosen setup $G_{v}$ is the unipotent radical of a Borel subgroup of $G$, hence Grosshans, and that $\mu_{V}: \overline{G \cdot v} \rightarrow \mathfrak{k}^{*}$ is not surjective.

Remark 2.10. If $N$ is the unipotent radical of a parabolic subgroup of $G$, a $G$-representation space $E$ containing $\bar{G} / N^{a}$ and a certain $K$-invariant hermitian inner product on $E$ are described in great detail in [Kir11], extending [GJS02] which dealt with unipotent radicals of Borel subgroups. In this situation it is natural to equip $\overline{G / N}^{\mathrm{a}}$ with the restriction of the associated flat Kähler form $\omega_{E}$ as above, since the associated symplectic structure coincides with the one obtained via symplectic implosion from the cotangent bundle $T^{*} K$.

\section{Hamiltonian G-extensions}

Let $G=K^{\mathbb{C}}$ be a complex reductive group with maximal compact subgroup $K$. Recall that a unipotent subgroup of $G$ is by definition an algebraic subgroup of $G$ consisting entirely of unipotent elements. Such groups are automatically nilpotent and connected, see [OV90, Chapter 3.2.2], hence simply-connected. Let $N$ be such a unipotent subgroup of $G$. Since our focus lies on actions of $N$, we suppose from now on that $G$ is connected and semisimple. Due to the simply-connectedness of $N$, by lifting to the universal cover if necessary, we may and will often assume that $G$ is simply-connected as well.

\section{A. Meromorphic actions and Hamiltonian extensions}

We will explore the relation between meromorphic $N$-actions and Hamiltonian $G$-actions.

Definition 3.1. Let $\left(X, \omega_{X}\right)$ be a connected compact Kähler manifold endowed with a holomorphic $N$ action. A Hamiltonian $G$-extension of (the $N$-action on) $\left(X, \omega_{X}\right)$ consists in a connected compact Hamiltonian $G$-manifold $\left(Z, \omega_{Z}\right)$ and an $N$-equivariant embedding $\iota: X \hookrightarrow Z$ such that for the de Rham cohomology classes associated with the Kähler forms we have

$$
\iota^{*}\left[\omega_{Z}\right]=\left[\omega_{X}\right]
$$

Remark 3.2. As $G=K^{\mathbb{C}}$, and hence $K$, is assumed to be semisimple, it follows from the fact that integration over $K$ does not change the cohomology class of a given Kähler form and from Remark 2.1 that any $N$-equivariant embedding of $\left(X, \omega_{X}\right)$ into a compact Kähler $G$-manifold $\left(Z, \omega_{Z}\right)$ satisfying Equation (3.1) is automatically a Hamiltonian $G$-extension.

The definition is motivated by the following example and the role it plays in the Geometric Invariant Theory of unipotent group actions on projective varieties, cf. [DK07, Section 5].

Example 3.3. Let $N$ act effectively on a smooth projective variety $X$. Any $N$-equivariant embedding $N \hookrightarrow \mathbb{P}(W)$, where $W$ is an $N$-representation space on which $N$ acts via an embedding $N \hookrightarrow \operatorname{SL}(W)$ is a Hamiltonian SL $(W)$-extension.

Example 3.4. Suppose that $G$ acts on $\left(X, \omega_{X}\right)$, extending the $N$-action. Since integration over $K$ does not change the cohomology class of $\omega_{X}$, we may, and will, suppose that $\omega_{X}$ is $K$-invariant. Therefore we can simply take $Z=X$ with $\iota=\mathrm{id}_{X}$ as Hamiltonian $G$-extension of the $N$-action on $X$. 
On the other hand, the twisted product ${ }^{3} G \times_{N} X$ is $G$-equivariantly isomorphic to $G / N \times X$ via the map $[g, x] \mapsto(g N, g \cdot x)$ and hence embeds into $Z:=\overline{G / N} \times X$ where $\overline{G / N}$ is a smooth projective $G$-equivariant compactification of the quasi-affine homogeneous space $G / N$. Endowing $Z$ with a direct product Kähler metric $\omega_{0} \oplus \omega_{X}$ and considering $\iota: X \hookrightarrow Z, \iota(x)=(e N, x)$, we obtain another Hamiltonian $G$-extension of the $N$-action on $\left(X, \omega_{X}\right)$.

Remark 3.5. Although the automorphism group of a compact Kähler manifold has a natural structure of a meromorphic group acting meromorphically on $X$, see [Fuj78, Theorem 5.5], this cannot be used in order to find a natural embedding of a unipotent algebraic group $N$ acting holomorphically on $X$ into a complex reductive group $G$ sitting inside $\operatorname{Aut}(X)$ as the following example shows.

Example 3.6. Consider the connected algebraic group

$$
G=\left\{\left(\begin{array}{ccc}
(a d-b c)^{-1} & z & w \\
0 & a & b \\
0 & c & d
\end{array}\right) ; a d-b c \neq 0\right\} \cong \mathrm{GL}(2, \mathbb{C}) \ltimes \mathbb{C}^{2} .
$$

According to [Br14, Theorem 1] there exists a 12-dimensional projective complex manifold $X$ having $\operatorname{Aut}^{0}(X)$ isomorphic to $G$.

The group

$$
N=\left\{\left(\begin{array}{ccc}
1 & 0 & z \\
0 & 1 & w \\
0 & 0 & 1
\end{array}\right) ; z, w \in \mathbb{C}\right\} \cong \mathbb{C}^{2}
$$

is a unipotent subgroup of $G$ which is not conjugate to a subgroup of the radical $R_{u}(G)$, nor to a subgroup of a Levi subgroup of $G$.

The group

$$
N=\left\{\left(\begin{array}{ccc}
e^{t} & t e^{t} & 0 \\
0 & e^{t} & 0 \\
0 & 0 & e^{-2 t}
\end{array}\right) ; t \in \mathbb{C}\right\} \cong \mathbb{C}
$$

is a non-algebraic subgroup of $G$. Consequently, $N$ does not act meromorphically on $X$. Its Zariski closure is the group

$$
\bar{N}=\left\{\left(\begin{array}{ccc}
t & s & 0 \\
0 & t & 0 \\
0 & 0 & t^{-2}
\end{array}\right) ; t \in \mathbb{C}^{*}, s \in \mathbb{C}\right\} \cong \mathbb{C}^{*} \times \mathbb{C} .
$$

Note that $\bar{N}$ and hence $N$ are not conjugate to subgroups of neither a Levi subgroup nor the radical of $G$.

Let us state the main result of this section.

Theorem 3.7. Let $N$ be a unipotent subgroup of the simply-connected complex semisimple Lie group G. For a connected compact Kähler manifold $\left(X, \omega_{X}\right)$ endowed with a holomorphic $N$-action the following statements are equivalent.

(1) There exists a Hamiltonian $G$-extension $\left(Z, \omega_{Z}\right)$ of the $N$-action on $X$.

(2) The $N$-action on $X$ is meromorphic.

(3) The twisted product $G \times_{N} X$ is Kähler.

Moreover, given a meromorphic $N$-action on $X$ we can always find a Hamiltonian $G$-extension that is a Gequivariant compactification of $G \times_{N} X$.

$3 \uparrow$ The twisted product $G \times_{N} X$ is by definition the quotient of $G \times X$ by the proper holomorphic $N$-action given by $n \bullet(g, x)=$ $\left(g n^{-1}, n \bullet x\right)$. The $N$-orbit of $(g, x)$ will be denoted by $[g, x] \in G \times_{N} X$. 
Remark 3.8. Since the centre of $G$ is finite and since $N$ does not contain finite subgroups, it follows that the $G$-action on $G \times_{N} X$ is effective whenever $N$ acts effectively on $X$.

Motivated by the above and by the equivalent conditions listed in Remark 2.2, we make the following definition that is central to our discussion.

Definition 3.9. Let $N$ be a unipotent subgroup of the simply-connected complex semisimple Lie group $G$, and let $X$ be a connected compact Kähler manifold endowed with a holomorphic $N$-action. We say that $\left(X, \omega_{X}\right)$ is a Hamiltonian $N$-manifold if there exists a Hamiltonian $G$-extension $\left(Z, \omega_{Z}\right)$ of the $N$-action on $X$.

The rest of this section is devoted to the proof of Theorem 3.7.

\section{B. Necessary conditions for the existence of a $G$-extension}

In this section, we will show the implications "(1) $\Rightarrow(3) \Rightarrow(2)$ " of Theorem 3.7. Let $\left(X, \omega_{X}\right)$ be a connected compact Kähler manifold on which $N$ acts holomorphically, and let $\alpha: X \rightarrow \operatorname{Alb}(\mathrm{X})$ be the natural map from $X$ to its Albanese torus.

Lemma 3.10. (“(1) $\Rightarrow(3) ")$ If the $N$-action on X admits a Hamiltonian G-extension, then the twisted product $G \times_{N} X$ is Kähler.

Proof. Let us consider the induced proper embedding $Y=G \times{ }_{N} X \hookrightarrow G \times{ }_{N} Z \cong G / N \times Z$. The claim follows from the fact $G / N$ is quasi-affine, and hence Kähler.

Remark 3.11. In fact, if $N$ is a connected nilpotent closed complex subgroup of $G$, then assuming that $G \times_{N} X$ be Kähler we can show that $N$ is in fact algebraic: applying [Bla56, Proposition II.1] to the fibre bundle $G \times_{N} X \rightarrow G / N$, we see that $G / N$ is Kähler, an application of [GMO11, Corollary 4.12] then yields the claim. This justifies our a priori algebraicity assumption on $N<G$.

Next, we embark on proving the implication "(3) $\Rightarrow(2)$ ". As a first step, we prove the following

Lemma 3.12. If $G \times_{N} X$ is Kähler, then the action of $N$ on the Albanese torus $\operatorname{Alb}(X)$ is trivial.

Proof. Suppose that the action of $N$ on $\operatorname{Alb}(X)$ is non-trivial. Then, as $N$ acts on $\operatorname{Alb}(X)$ by translations, we can find a closed one-parameter subgroup $\mathbb{C} \hookrightarrow N$ such that $\operatorname{dim} \mathbb{C} \cdot[0]=1$ where $[0]$ denotes the base point of $\operatorname{Alb}(X)$. The topological closure $T:=\overline{\mathbb{C} \cdot[0]} \subset \alpha(X) \subset \operatorname{Alb}(X)$ is a connected compact subgroup of $\operatorname{Alb}(X)$, hence a subtorus. Its pre-image $\alpha^{-1}(T)$ is a compact $\mathbb{C}$-invariant subvariety of $X$. According to the Jacobson-Morozov Theorem, see [Jac62, Theorem III.17], there is a closed subgroup $S$ of $G$ that is locally isomorphic to $\operatorname{SL}(2, \mathbb{C})$ and contains the one-parameter subgroup $\mathbb{C} \hookrightarrow N$ under discussion. Since $G \times_{N} X$ is Kähler and contains $Y^{\prime}:=S \times_{\mathbb{C}} \alpha^{-1}(T)$ as a closed $S$-invariant analytic subset, every $S$-orbit in $Y^{\prime}$ and therefore every $\mathbb{C}$-orbit in $\alpha^{-1}(T)$ is Zariski-open in its closure by [GMO11, Corollary 3.9].

There exists a 1 -dimensional orbit $\mathbb{C} \cdot x$ in $X$ such that $\alpha(x)=[0] \in \operatorname{Alb}(X)$. Consequently, going through the possible stabiliser subgroups, we see that there are two possibilities: either the orbit $\mathbb{C} \cdot x$ is compact, i.e., it is a 1-dimensional complex torus, or the normalisation of $\overline{\mathbb{C} \cdot x}$ is biholomorphic to $\mathbb{P}_{1}$. Both cases lead to contradictions. Indeed, in the first case, the isotropy of $\mathbb{C}$ and hence the isotropy in $S$ would be infinite discrete, which contradicts [GMO11, Proposition 4.4], while in the second case the fact that $\left.\alpha\right|_{\mathbb{C} \cdot x}$ is non-constant by construction would produce a non-zero holomorphic 1 -form on $\mathbb{P}^{1}$.

We remark that the fact that $N$ acts trivially on $\operatorname{Alb}(X)$ alone does not imply the existence of a Hamiltonian $G$-extension of the $N$-action on $X$ as the following example shows. 
Example 3.13. Let $N=\left(\begin{array}{ll}1 & \mathbb{C} \\ 0 & 1\end{array}\right) \subset G=\operatorname{SL}(2, \mathbb{C})$ and consider its action on $X=\mathbb{P}_{2}$ given by

$$
t \cdot\left[x_{0}: x_{1}: x_{2}\right]:=\left[e^{t} x_{0}: e^{i t} x_{1}: x_{2}\right]
$$

The $N$-orbits in $X$ are not locally Zariski closed, and there are elements having isotropy isomorphic to $\mathbb{Z}$. According to [GMO11, Theorem 3.6] $G \times_{N} X$ cannot be Kähler. Hence, there does not exist a Hamiltonian $G$-extension of the $N$-action on $X$ although $N$ acts trivially on $\operatorname{Alb}(X)=\{\mathrm{pt}\}$.

Our next goal is to show that, if there exists a Hamiltonian $G$-extension of the $N$-action on $X$, then $N$ acts meromorphically on $X$. Due to Lemma 3.12 the claim is equivalent to the fact that the map $N \rightarrow \operatorname{Aut}_{\mathrm{aff}}(X)$ induced by the action has analytically Zariski-closed image, where $\operatorname{Aut}_{\text {aff }}(X)$ denotes the kernel of the Jacobi homomorphism $\alpha_{*}: \operatorname{Aut}^{0}(X) \rightarrow \operatorname{Aut}^{0}(\operatorname{Alb}(X))$, cf. [Fuj78, §2]. Since by [Fuj78, Lemma 4.6 and Theorem 5.5] or [Lie78, Proposition 2.1] the analytic Zariski-topology on the meromorphic subgroup $\operatorname{Aut}_{\text {aff }}(X)<\operatorname{Aut}^{0}(X)$ is obtained from the complex structure on the cycle space $\mathscr{C}_{\operatorname{dim} X}(X \times X)$, as a first step we prove a technical lemma about induced actions on cycle spaces.

Lemma 3.14. Let $N$ be a unipotent subgroup of $G$, let $M$ be a Kähler manifold endowed with a holomorphic $N$-action, and let $\mathscr{C}_{k}(M)$ be the Barlet space of compact $k$-cycles. Then, there exists a natural $G$-equivariant isomorphism $G \times_{N} \mathscr{C}_{k}(M) \cong \mathscr{C}_{k}\left(G \times_{N} M\right)$.

Proof. For every irreducible compact analytic subset $A$ of $M$ and each $g \in N$, the image $g(A)$ is again an irreducible compact analytic subset of $M$. Hence, we obtain a holomorphic $N$-action on $\mathscr{C}_{k}(M)$ by the obvious extension of this action to $k$-cycles. Moreover, the inclusion $\iota: M \rightarrow G \times_{N} M, x \mapsto[e, x]$ induces a proper holomorphic embedding of $\mathscr{C}_{k}(M)$ into $\mathscr{C}_{k}\left(G \times_{N} M\right)$ by sending $A \in \mathscr{C}_{k}(M)$ to $\iota(A)$. From this we obtain a well-defined injective and immersive holomorphic map

$$
\Phi_{k}: G \times_{N} \mathscr{C}_{k}(M) \hookrightarrow \mathscr{C}_{k}\left(G \times_{N} M\right), \quad \Phi_{k}([g, A]):=g \cdot \iota(A) .
$$

Suppose for a moment that $A \subset G \times_{N} M$ is an irreducible compact analytic subset. Since $G / N$ is quasiaffine, and hence in particular holomorphically separable, the bundle projection $\pi: G \times{ }_{N} X \rightarrow G / N$ maps $A$ to a point $g N \in G / N$. Consequently, $\mathscr{C}_{k}\left(G \times_{N} M\right)$ coincides with the space $\mathscr{C}_{k}\left(G \times_{N} M\right)_{\pi}$ of $\pi$-relative cycles. Moreover, the natural $G$-action on $\mathscr{C}_{k}\left(G \times_{N} M\right)$ makes the resulting projection $\mathscr{C}_{k}\left(G \times_{N} M\right) \rightarrow G / N$ equivariant.

We hence conclude from the fact that $\Phi_{k}$ induces an isomorphism of the fibres over $e N$ that the image of $\Phi_{k}$ is all of $\mathscr{C}_{k}\left(G \times_{N} M\right)_{\pi}=\mathscr{C}_{k}\left(G \times_{N} M\right)$. The claim follows.

Proposition 3.15. (“(3) $\Rightarrow(2) ”)$ If $G \times_{N} X$ is Kähler, then $N$ acts meromorphically on $X$.

Proof. Composed with the the natural embedding $\operatorname{Aut}_{\text {aff }}(X) \hookrightarrow \mathscr{C}_{n}(X \times X)$, the action map $N \rightarrow$ Aut $_{\text {aff }}(X)$ yields the following holomorphic map:

$$
\begin{aligned}
\iota: N & \rightarrow \mathscr{C}_{n}(X \times X) \\
g & \mapsto \Gamma_{x \mapsto g} \bullet x:=\{(x, g \cdot x) \mid x \in X\} .
\end{aligned}
$$

We set $n:=\operatorname{dim} X$ and consider the $N$-action on the product $X \times X$ given by $g \cdot(x, y)=(x, g \cdot y)$. We have $G \times_{N}(X \times X) \cong\left(G \times_{N} X\right) \times X$, which shows that $G \times_{N}(X \times X)$ is Kähler. Hence, the cycle space $\mathscr{C}_{n}\left(G \times \times_{N}(X \times X)\right)$ is Kähler by [BV89, Théorème 2]. Applying Lemma 3.14 to $M=N \times N$ we infer that $G \times{ }_{N} \mathscr{C}_{n}(X \times X)$ is likewise Kähler. It therefore follows from [GMO11, Theorem 3.6] that all $N$-orbits in $\mathscr{C}_{n}(X \times X)$ are locally closed in the analytic Zariski-topology.

Now, we notice that $\iota(N)$ coincides with the $N$-orbit of $\Delta_{X} \in \mathscr{C}_{n}(X \times X)$, where $\Delta_{X}$ denotes the diagonal in $X \times X$. This implies that $\iota(N)$ is a locally Zariski-closed, hence Zariski-closed subgroup of $\operatorname{Aut}_{\text {aff }}(X)<\operatorname{Aut}^{0}(X) \subset \mathscr{C}_{n}(X \times X)$, cf. [Hum75, Section 7.4], as was to be shown. 
For later usage, we note the following, related observation.

Lemma 3.16. An algebraic subgroup $H$ of $G$ which acts meromorphically on $X$ acts automatically trivially on $\operatorname{Alb}(X)$.

Proof. This follows from the fact that every meromorphic homomorphism from an affine linear group to a compact complex torus is constant, see [Fuj78, Lemma 3.8].

\section{C. Existence of a Hamiltonian G-extension}

In this section, we will show the crucial implication "(2) $\Rightarrow(1)$ " of Theorem 3.7, i.e., the fact that meromorphic actions of unipotent algebraic subgroups of semisimple groups $G$ always admit Hamiltonian $G$ extensions, thereby completing the proof.

Let us recall the setup: Let $N$ be a unipotent subgroup of the simply-connected complex semisimple Lie group $G$, and let $X$ be a connected compact Kähler manifold endowed with a meromorphic $N$-action.

\section{C.a. The case of unipotent radicals of Borel subgroups}

Let $B$ be a Borel subgroup of $G$ having Levi decomposition $B=T U=U T$. In a first step, we make the additional assumption that $N$ coincides with the the unipotent radical $U$ of $B$; i.e., $N=U$.

Let us consider the twisted product $M:=B \times_{U} X$ and the $U$-equivariant inclusion $\iota_{X}: X \hookrightarrow M$ as the fibre over $e U$. Since the principal bundle $B \rightarrow B / U \cong T$ is holomorphically trivial, the same holds for the associated fibre bundle; i.e., we have $M \cong T \times X$. Explicitly, an isomorphism $B \times_{U} X \rightarrow T \times X$ is given by $[t u, x] \mapsto(t, u \bullet x)$. A direct calculation shows that the induced $B$-action on $T \times X$ is given by the formula

$$
(t u) \cdot(s, x)=\left(t s,\left(s^{-1} u s\right) \cdot x\right) .
$$

Let $\bar{G}$ be a projective meromorphic structure on $G$. Since the subgroups $T, U$ and $B$ are algebraic in $G$, their topological closures in $\bar{G}$ are analytic.

Lemma 3.17. If $U$ acts meromorphically on $X$, then the B-action on $T \times X$ defined in Equation (3.2) extends to a meromorphic B-action on $\bar{T} \times X$. In particular, there exists a B-equivariant Kähler compactification $\left(\bar{M}, \omega_{\bar{M}}\right)$ of $M \supset \iota_{X}(X)$ such that $\left[\iota_{X}^{*}\left(\omega_{\bar{M}}\right)\right]=\left[\omega_{Z}\right]$.

Proof. By the definition of a meromorphic structure the map $c: T \times U \rightarrow U,(s, u) \mapsto s^{-1} u s$, extends to a meromorphic map $\bar{c}: \bar{T} \times \bar{U} \rightarrow \bar{U}$ which is holomorphic on $(\bar{T} \times U) \cup(T \times \bar{U})$. Analogously, for the same reason the multiplication map $m: T \times T \rightarrow T$ extend meromorphically to $\bar{T} \times \bar{T} \rightarrow \bar{T}$ making $\bar{T}$ a $T$-bi-equivariant compactification of $T$. Denote the extended map by $\bar{m}$. With this notation, $B$ acts on $\bar{T} \times X$ by the formula

$$
(t u) \cdot(s, x):=(\bar{m}(t, s), \bar{\alpha}(\bar{c}(s, u), x)),
$$

where $\bar{\alpha}: \bar{U} \times X \rightarrow X$ is the meromorphic extension of the $U$-action on $X$. It is hence clear that $B$ acts meromorphically on $\bar{T} \times X$.

In the next step we consider the holomorphic fibre bundle $Z:=G \times_{B} \bar{M} \stackrel{\pi}{\longrightarrow} G / B$. The natural inclusion $\bar{M} \hookrightarrow G \times \times_{B} \bar{M}$ as the fibre over $e B$ is denoted by $\iota_{\bar{M}}$. Both the typical fibre $\bar{M}$ and the base $G / B$ of $Z$ are Kähler. Using Blanchard's theorem [Bla56, Théorème principal II], we will construct a Kähler form on $Z$ such that $Z$ is a Hamiltonian $G$-extension of the $U$-action on $X$.

Since $G / B$ is simply-connected, in order to be able to apply Blanchard's result and hence show that $G \times{ }_{B} \bar{M}$ is Kähler, we only have to check that the transgression map from $H^{1}(\bar{M}, \mathbb{R})$ to $H^{2}(G / B, \mathbb{R})$ is identically zero. Recall that this map is defined on the set of transgressive elements in $H^{1}(\bar{M}, \mathbb{R})$ in the following way. A class $[\alpha] \in H^{1}(\bar{M}, \mathbb{R})$ is called transgressive if there exists a 1 -form $\beta$ on $Z$ such that $\left[\iota_{\bar{M}}^{*} \beta\right]=[\alpha]$ and such that there exists a 2 -form $\tau$ on $G / B$ such that $d \beta=\pi^{*} \tau$. Since $\pi^{*}$ is injective, we 
have $d \tau=0$, and the transgression map then associates $[\tau] \in H^{2}(G / B, \mathbb{R})$ to $[\alpha] \in H^{1}(\bar{M}, \mathbb{R})$, see $[\mathrm{BT} 82$, Proposition 18.13]. As a side-remark we note that this transgression map is zero if and only if $b_{1}(Z)=$ $b_{1}(G / B)+b_{1}(\bar{M})$ where $b_{1}$ denotes the first Betti number, see [Bla56, p. 192].

Lemma 3.18. Suppose that $B$ acts trivially on $\operatorname{Alb}(\bar{M})$. Then, every $[\alpha] \in H^{1}(\bar{M}, \mathbb{R})$ is transgressive, and the transgression map is identically zero.

Proof. As $\bar{M}$ is Kähler by Lemma 3.17, we may use the Hodge decomposition to write

$$
[\alpha] \in H^{1}(\bar{M}, \mathbb{R}) \subset H^{1}(\bar{M}, \mathbb{C})=H^{1,0}(\bar{M}) \oplus \overline{H^{1,0}(\bar{M})}=H^{0}\left(\Omega \frac{1}{M}\right) \oplus \overline{H^{0}\left(\Omega \frac{1}{M}\right)}
$$

as $[\alpha]=\eta+\bar{\eta}$ where $\eta$ is a holomorphic 1 -form on $\bar{M}$. Since by hypothesis and Lemma 3.16 the algebraic subgroup $B<G$ acts trivially on $\operatorname{Alb}(\bar{M})$, the form $\eta$ is $B$-invariant, hence extends to a $G$-invariant holomorphic 1 -form $\hat{\eta} \in H^{0}\left(\Omega_{Z}^{1}\right)^{G}$. Since $d \hat{\eta}$ is likewise $G$-invariant, it is uniquely determined by its restriction $\iota_{\bar{M}}^{*}(d \hat{\eta})=d\left(\iota_{\bar{M}}^{*} \hat{\eta}\right)=d \eta=0$. Consequently, $d \hat{\eta}=0=\pi^{*} \mathbf{0}$. We conclude that $[\alpha]$ is transgressive and is mapped to $\mathbf{0}+\mathbf{0}=\mathbf{0} \in H^{2}(G / B, \mathbb{R})$ by the transgression map.

Remark 3.19. The proof works more generally for a parabolic subgroup $P<G$ acting on a compact Kähler manifold such that the induced action on the Albanese is trivial. To be more precise, we see that for every such $P$-manifold $X$ the twisted product $G \times_{P} X$ is Kähler. As the $G$-action on this manifold is Hamiltonian, by applying [GMO11, Theorem 3.6] or [Fuj96, Remark after Lemma 2.1] together with [Fuj78, Proposition 6.10] we conclude that every $P$-orbit in $X$ is Zariski-open in its closure and that the $P$-action on $X$ is meromorphic. This generalises and gives a new proof for a result of Sommese, cf. [Som75, §3]. For criteria guaranteeing triviality of induced actions on Albanese tori see [Fuj78, §6c)].

Combining Lemmata 3.17, 3.16, and 3.18 with Blanchard's theorem we conclude that the twisted product $Z=G \times{ }_{B} \bar{M}$ is Kähler. Moreover, the first step of Blanchard's proof [Bla56, p. 187] shows that the cohomology class of the constructed Kähler form on $Z=G \times_{B} \bar{M}$ pulls back under $\iota_{\bar{M}}$ to $\left[\omega_{\bar{M}}\right]$ on $\bar{M}$. Embedding $X$ into $\bar{M}$ by $\iota_{X}$ and further into $Z$ by $\iota_{\bar{M}}$ we obtain a Hamiltonian $G$-extension of the $U$-action on $X$. Notice that $Z$ contains $G \times_{U} X \cong G \times_{B}\left(B \times_{U} X\right)$ as a Zariski open subset. In fact, $Z=G \times_{B} \bar{M} \cong G \times{ }_{B}(\bar{T} \times X) \rightarrow G \times_{B} \bar{T}$ is an extension of the $X$-fibre bundle $G \times_{U} X \rightarrow G / N$ to the projective completion $G \times{ }_{B} \bar{T}$ of $G / N$.

\section{C.b. The general case}

In the second and final step we show that the case of an arbitrary unipotent subgroup $N$ of $G$ can be reduced to the unipotent radical $U$ of a Borel subgroup $B \subset G$.

Lemma 3.20. Let $N$ be a unipotent subgroup of $G$. Then there exists a Borel subgroup $B=T U$ of $G$ such that $N \subset U$. In addition, if $N$ acts effectively and meromorphically on $X$, then $U \times_{N} X$ admits a $U$-equivariant Kähler compactification on which $U$ acts effectively and meromorphically and whose Kähler class extends the given Kähler class $\left[\omega_{X}\right]$ on X.

Proof. The first statement is [Hum75, 30.4, Theorem]. Moreover, from the first paragraph in the proof of [Akh95, Chapter 5, Theorem 4] we conclude that the $N$-principal bundle $U \rightarrow U / N$ admits an algebraic section, whose image we call $S$. As a consequence, we obtain an $N$-equivariant isomorphism $N \times S \rightarrow U$ with $S \cong U / N$. Let $\bar{S}$ be a $U$-equivariant smooth projective compactification of $S$. Following the line of argumentation presented at the beginning of Section 3.C.c we conclude as before that $\bar{S} \times X$ is a compact Kähler manifold endowed with a meromorphic $U$-action, containing $U \times_{N} X$ as a Zariski-open set. Again, we can choose the Kähler form on $\bar{S} \times X$ such that its class extends the given Kähler class $\left[\omega_{X}\right]$.

Finally, by applying the discussion of Section 3.C.c to the compact Kähler manifold $X^{\prime}=\bar{S} \times X$ with meromorphic $U$-action obtained in Lemma 3.20 and noticing that

$$
Y=G \times_{N} X \cong G \times_{B}\left(B \times_{U}\left(U \times_{N} X\right)\right) \cong G \times_{B}(T \times(U / N) \times X)
$$

we arrive at 
Proposition 3.21. (“(2) $\Rightarrow(1) ")$ Let $N$ be a unipotent subgroup of $G$ acting effectively and meromorphically on the compact Kähler manifold X. Then, there exists a Hamiltonian $G$-extension $Z$ of the $N$-action on $X$, which can be chosen such that it contains $Y:=G \times_{N} X$ as a Zariski open subset. More precisely, we might choose

$$
Z=\bar{Y}=G \times{ }_{B}(\bar{T} \times \overline{U / N} \times X),
$$

where $B=T U$ is a Borel subgroup of $G$ with $N \subset U$ and where $\bar{T} \times \overline{U / N}$ is the B-equivariant compactification of $T \times(U / N)$ constructed in Lemmata 3.17 and 3.20.

Remark 3.22. (Non-uniqueness) There are many possible ways to choose the compactifications $\bar{T}$ and $\bar{S}$; in the first case, we have all smooth projective toric varieties of $\operatorname{dimension} \operatorname{dim} T$ to choose from.

\section{C.c. Additional observations}

We note a minimality property of the above construction that is crucial for subsequent arguments on the independence of the set of semistable points from the chosen G-extensions, see Section 4.B below.

Proposition 3.23. Let $X$ be a compact Hamiltonian $N$-manifold and let $\bar{Y}$ be the $G$-equivariant compactification of $Y:=G \times_{N} X$ whose existence is guaranteed by Proposition 3.21. Then, for any Hamiltonian G-extension $Z$ of the $N$-action on $X$, there exists a G-equivariant embedding $\bar{Y} \hookrightarrow \overline{G / N} \times Z$ where $\overline{G / N}$ is a certain $G$-equivariant compactification of $G / N$.

Proof. Let $Z$ be any $G$-extension of the $N$-action on $X$. The $N$-equivariant embedding $X \hookrightarrow Z$ induces a G-equivariant embedding $Y=G \times_{N} X \hookrightarrow G \times_{N} Z \cong G / N \times Z$. The identification $G / N \cong G \times{ }_{B}(T \times U / N)$, cf. Equation (3.3), suggests choosing the compactified fibre bundle

$$
\overline{G / N}:=G \times \times_{B}(\bar{T} \times \overline{U / N})
$$

as a well-adapted $G$-equivariant compactification of $G / N$. With this definition we obtain the desired embedding $\bar{Y}=G \times_{B}(\bar{T} \times \overline{U / N} \times X) \hookrightarrow G \times_{B}(\bar{T} \times \overline{U / N} \times Z) \cong \overline{G / N} \times Z$.

Remark 3.24. Since $N$ is a connected subgroup of the simply-connected group $G$, the quasi-affine variety $G / N$ is simply-connected. Consequently, every smooth compactification of $G / N$ is likewise simplyconnected, see [FL81, 0.7(B)]; in particular, this observation applies to the compactification constructed in Proposition 3.23.

Example 3.25. Suppose that $N=\left(\begin{array}{ll}1 & \mathbb{C} \\ 0 & 1\end{array}\right) \subset G=\operatorname{SL}(2, \mathbb{C})$. Choosing $\bar{T}=\mathbb{P}_{1}$, we see that $\overline{G / N}=G \times_{B} \bar{T}$ is the blow-up of $\mathbb{P}_{2}$ at the origin, i.e., the first Hirzebruch surface.

\section{The set of semistable points with respect to unipotent groups}

Let $G=K^{\mathbb{C}}$ be a simply-connected semisimple complex Lie group with maximal compact subgroup $K$, let $N$ be a unipotent subgroup of $G$, and let $\left(X, \omega_{X}\right)$ be a compact Hamiltonian $N$-manifold. In this section we explain how one can use the concept of Hamiltonian $G$-extensions in order to define the set of semistable points $X_{N}^{s S}\left[\omega_{X}\right]$ for the $N$-action on $X$.

\section{A. Defining the set of semistable points}

We will slowly approach the goal of defining the correct notion of semistability, see Definition 4.2 below. 


\section{A.a. The naive approach}

Since $\left(X, \omega_{X}\right)$ is a compact Hamiltonian $N$-manifold, we can find a Hamiltonian $G$-extension $\left(Z, \omega_{Z}\right)$ of the $N$-action on $X$ with $N$-equivariant embedding $\iota: X \hookrightarrow Z$. It is tempting to define the set of $N$-semistable points in $X$ as the $N$-invariant open subset $l^{-1}\left(Z_{G}^{s s}\left[\omega_{Z}\right]\right)$. As the following example shows, this set heavily depends on the choice of the $G$-extension $\left(Z, \omega_{Z}\right)$.

Example 4.1. Let $X=\mathbb{P}_{1}$ with the Fubini-Study metric $\omega_{F S}$ and let $N=\mathbb{C}$ act on $\mathbb{P}_{1}$ by $t \cdot\left[x_{0}: x_{1}\right]=$ $\left[x_{0}+t x_{1}: x_{1}\right]$. Since the $N$-action extends to an action of $G=\operatorname{SL}(2, \mathbb{C})$, we have the following three Hamiltonian $G$-extensions.

(1) $Z_{1}=X$ where $\iota_{1}=\mathrm{id}_{X}$,

(2) $Z_{2}=X \times X$ endowed with $\omega_{Z_{2}}=\frac{1}{2}\left(\omega_{F S} \oplus \omega_{F S}\right)$ and the diagonal $G$-action, where the embedding is given by $\iota_{2}(x)=(x, x)$, and

(3) $Z_{3}=Z_{2}$ with $\omega_{Z_{3}}=2 \omega_{Z_{2}}$ and with the diagonal $G$-action where $\iota_{3}(x)=([1: 0], x)$.

It is not hard to show that $\iota_{1}^{-1}\left(Z_{1, G}^{s S}\right)=\iota_{2}^{-1}\left(Z_{2, G}^{s S}\right)=\emptyset$ while $\iota_{3}^{-1}\left(Z_{3, G}^{s S}\right)=\mathbb{P}_{1} \backslash\{[1: 0]\}$.

\section{A.b. The case of linear actions on projective manifolds}

Let us recall the approach taken by Doran and Kirwan in [DK07]. There, the authors study the situation where $X$ is a subvariety of $\mathbb{P}(W)$ for some finite-dimensional $G$-module $W$ and they consider the embedding $\hat{\imath}: G \times_{N} X \hookrightarrow G \times_{N} \mathbb{P}(W) \cong(G / N) \times \mathbb{P}(W)$ that is induced by the embedding $\iota: X \hookrightarrow \mathbb{P}(W)$. Then, they equip $G \times_{N} X$ with the $G$-linearised ample line bundle $L:=\hat{\iota}^{*}\left(\operatorname{pr}_{1}^{*}\left(\mathscr{O}_{G / N}\right) \otimes \operatorname{pr}_{2}^{*}\left(\mathscr{O}_{\mathbb{P}(W)}(1)\right)\right)$. In this way, Doran and Kirwan can consider the set of Mumford-semistable points $\left(G \times_{N} X\right)_{G}^{s S}(L)$ in $G \times_{N} X$ and they proceed by defining the set of Mumford-semistable points in $X$ as $X_{N}^{s S}(L):=\iota^{-1}\left(G \times_{N} X\right)_{G}^{s S}(L)$, see [DK07, Definition 5.1.5]. As shown in [DK07, Propositions 5.1.8 and 5.1.9], this set $X_{N}^{s S}$ does not depend on the choice of the group $G$ and can be intrinsically defined knowing only the $N$-action on $X$.

\section{A.c. The definition of semistability}

The algebraic approach suggests that in our situation, we should choose a Hamiltonian $G$-extension $\left(Z, \omega_{Z}\right)$ of the $N$-action on $X$ and then consider the analogous embedding $Y:=G \times_{N} X \hookrightarrow G \times_{N} Z \cong G / N \times Z$, such that the Hamiltonian $G$-extension $Z$ plays the role of the projectivised $G$-module $\mathbb{P}(W)$.

To implement this idea, first note that the $N$-equivariant embedding $\iota: X \hookrightarrow Z$ induces the $G$ equivariant proper embedding

$$
\hat{\imath}: Y=G \times_{N} X \hookrightarrow G \times_{N} Z \cong G / N \times Z
$$

given by $\hat{\imath}([g, x])=(g N, g \bullet \iota(x))$. Secondly, recalling that $G / N$ is quasi-affine, choose a $G$-representation space $V$ containing $G / N$ as an orbit as well as a $K$-invariant hermitian inner product on $V$, which induces $K$-invariant Kähler forms $\omega_{V}$ on $G / N$ and

$$
\hat{\omega}_{Z}:=\omega_{V} \oplus \omega_{Z}
$$

on $G / N \times Z$. Then, we take

$$
\omega_{Y}:=\hat{\iota}^{*} \hat{\omega}_{Z}
$$

as Kähler form on $Y$. The spaces considered so far fit into following commutative diagram

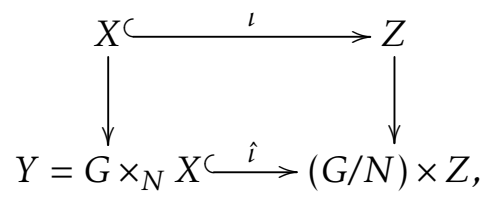


where the vertical arrows correspond to the maps given by $x \mapsto[e, x]$ and $z \mapsto(e N, z)$, respectively. It follows that $\left[\omega_{Y}\right]$ extends $\left[\omega_{X}\right]$ to $Y$. Recall that, as $K$ is semisimple and $\omega_{Y}$ is $K$-invariant, there exists a unique moment map $\mu_{Y}: Y \rightarrow \mathrm{k}^{*}$ for the $K$-action on $Y$, see Remark 2.1; in other words, $Y$ is a (noncompact) Hamiltonian $G$-manifold. We will use $\mu_{Y}$ to define a notion of semistability for the $N$-action:

Definition 4.2. Let $\left(X, \omega_{X}\right)$ be a Hamiltonian $N$-manifold. We define the set of $N$-semistable points (with respect to $\left.\left[\omega_{X}\right]\right)$ as

$$
X_{N}^{s S}\left[\omega_{X}\right]:=X \cap Y_{G}^{s S}\left[\omega_{Y}\right],
$$

where the Kähler form $\omega_{Y}$ on $Y=G \times_{N} X$ is given by Equations (4.2) and (4.3) above. Analogously, the set of $N$-stable points in $X$ is defined as $X_{N}^{s}\left[\omega_{X}\right]:=X \cap Y_{G}^{s}\left[\omega_{Y}\right]$, where we set $Y_{G}^{s}\left[\omega_{Y}\right]:=G \bullet \mu_{Y}^{-1}(0){ }^{4}$

Note that $X_{N}^{S S}\left[\omega_{X}\right]$ a priori depends on the choice of $G, \omega_{V}$, and $Z$ although we do not convey this information in our notation. We will discuss the choices regarding $G$ and the metric on $G / N$ in Section 4.C below. As we will see, the definition is actually independent of the choice of a Hamiltonian $G$-extension, once $G$ and the metric on $G / N$ are fixed; see the subsequent section.

Example 4.3. (A non-projective compact Kähler manifold with meromorphic $\mathbb{C}$-action) Let $S$ be a non-projective $K 3$-surface with $\operatorname{Pic}(S) \neq\{e\}$, and let $L \rightarrow S$ be a non-trivial holomorphic line bundle on $S$ with zero section $Z_{L} \subset L$. Let $P:=L \backslash Z_{L} \rightarrow S$ be the associated $\mathbb{C}^{*}$-principal bundle, and consider the non-trivial $\mathbb{P}^{1}$-bundle

$$
M:=P \times_{\mathbb{C}^{*}} \mathbb{P}^{1} \longrightarrow P / \mathbb{C}^{*}=S .
$$

As the Albanese of $\mathbb{P}^{1}$ is trivial and since $S$ is a simply-connected compact Kähler manifold, the compact complex manifold $M$ is Kähler by Blanchard's theorem, cf. the discussion in Section 3.C.c. On the other hand, as the non-projective surface $S$ embeds into $M$ as zero section, $Y$ is likewise non-projective. Since the corresponding vector field has zeroes, the effective $\mathbb{C}^{*}$-action from the left is trivial on the Albanese of $M$, see [Fuj78, Prop. 6.8] or [Lie78, Proposition 3.14]. Trivially extend the action of $\mathbb{C}^{*}$ on $M$ to an action of the Borel subgroup $\mathbb{C}^{*}<B$ of lower-triangular matrices in $\operatorname{SL}(2, \mathbb{C})=: G$, and define

$$
X:=G \times{ }_{B} M .
$$

Since the $B$-action on the Albanese of $M$ is trivial and since $\mathbb{P}^{1}$ is simply-connected, it again follows from Blanchard's theorem that the compact complex manifold $X$ is Kähler, see also Lemma 3.18. Moreover, the $G$-action on $X$ induces an action of the unipotent algebraic subgroup $N<G$ of strictly upper-triangular elements of $\operatorname{SL}(2, \mathbb{C})$. Set $K:=\mathrm{SU}(2)$. Then, any $K$-invariant Kähler form $\omega_{X}$ on $X$ produces a moment map $\mu_{X}: X \rightarrow \mathfrak{k}^{*}$ for the $K$-action; i.e., $X$ is its own Hamiltonian $G$-extension, so that the $N$-action on $X$ is meromorphic.

Now, apply the construction described at the beginning of the present section to $X$. As $G>N$ already acts on $X$, we can choose $Z=X$ as Hamiltonian $G$-extension. Let $\omega_{V}$ be the essentially unique flat $K$ invariant Kähler form on $G / N \cong \mathbb{C}^{2} \backslash\{0\} \subset V=\mathbb{C}^{2}$, and hence consider the Kähler form $\omega_{Y}=\omega_{V} \oplus \omega_{X}$ on $Y:=G \times_{N} X \cong G / N \times X$, together with the resulting moment map $\mu_{Y}=\mu_{G / N}+\mu_{X}: Y \rightarrow \mathfrak{k}^{*}$. We claim that $X_{N}^{s s}\left[\omega_{X}\right] \neq \emptyset$, which will conclude our construction. In order to establish the claim, we first note that $\mu_{X}(X) \neq\{0\}$, as otherwise Equation (2.2) would imply that the $K$-action on $X$ is trivial. Let $\beta_{0} \in \mu_{X}(X) \backslash\{0\}$ and choose $x_{0} \in \mu_{X}^{-1}\left(\beta_{0}\right)$. We have $\mu_{G / N}(G / N)=\mathfrak{k}^{*} \backslash\{0\}$, cf. Section 2.D, and hence there exists $g_{0} \in G$ such that $\mu_{G / N}\left(g_{0} U\right)=-\beta_{0}$. By construction, we then have $\mu_{Y}\left(g_{0} \bullet\left(e U, g_{0}^{-1} \cdot x_{0}\right)\right)=\mu_{Y}\left(g_{0} U, x_{0}\right)=0$, and hence $\left(e U, g_{0}^{-1} \bullet x_{0}\right) \in Y_{G}^{s s}\left[\omega_{Y}\right] \cap(\{e U\} \times X)=X_{N}^{s s}\left[\omega_{X}\right]$, which is therefore non-empty, as claimed.

$4 \uparrow$ With this definition, a semistable point $x \in X$ is $N$-stable if and only if $N \bullet x$ is closed in $X_{N}^{s S}\left[\omega_{X}\right]$. In view of Lemma 5.1 and Theorem 5.3 below this is the correct notion in our situation. The reader should however be aware that there are several notions of (proper) stability in use in the literature. 


\section{B. Semistability does not depend on the $G$-extension}

In contrast to the naive definition of semistability discussed in Section 4.A.a above, it turns out the set of $G$-semistable points in $Y$ with respect to $\omega_{Y}$ as defined in Definition 4.2 does not depend on the choice of the Hamiltonian $G$-extension $\left(Z, \omega_{Z}\right)$.

Theorem 4.4. Let $N$ be a unipotent subgroup of the simply-connected semisimple complex Lie group $G$, acting meromorphically on the compact Kähler manifold $\left(X, \omega_{X}\right)$. Let $\left(Z_{j}, \omega_{j}\right), j=1,2$, be two Hamiltonian $G$ extensions of the $N$-action on $X$. Choose a $G$-equivariant algebraic embedding $G / N \hookrightarrow V$ into a $G$-representation space $V$, and a $K$-invariant Hermitian inner product on $V$, inducing Kähler forms $\omega_{V}$ on $G / N, \hat{\omega}_{Z}$ on $G / N \times Z$, and $\omega_{Y, 1}, \omega_{Y, 2}$ on $Y$, as described in Section 4.A.a. Then, we have $Y_{G}^{s S}\left[\omega_{Y, 1}\right]=Y_{G}^{s S}\left[\omega_{Y, 2}\right]$.

Proof. We will prove that on the smooth Kähler compactification $\bar{Y}$ of $Y$ constructed in Proposition 3.21 there exists a $(1,1)$-form $\alpha \in \mathcal{A}^{1,1}(\bar{Y})$ satisfying the following two properties:

(a) $\left.\alpha\right|_{Y}=\omega_{Y, 2}-\omega_{Y, 1}$, and

(b) $[\alpha]=0 \in H^{2}(\bar{Y}, \mathbb{R})$.

It then follows from the $\partial \bar{\partial}$-lemma on the compact Kähler manifold $\bar{Y}$ that there is a smooth function $\varphi \in \mathcal{C}^{\infty}(\bar{Y})$ such that $\alpha=i \partial \bar{\partial} \varphi$. Restricting everything to $Y$, we obtain a bounded smooth function $\varphi$ on $Y$ such that $\omega_{Y, 2}=\omega_{Y, 1}+i \partial \bar{\partial} \varphi$. In this situation, we can repeat the proof of Proposition 2.4 to deduce $Y_{G}^{s s}\left[\omega_{Y, 1}\right]=Y_{G}^{s s}\left[\omega_{Y, 2}\right]$. Hence, in order to complete the argument, we must show existence of $\bar{Y}$ and $\alpha$ with the above properties.

To do so, we note first that $\left(Z, \omega_{Z}\right):=\left(Z_{1} \times Z_{2}, \frac{1}{2}\left(\omega_{1} \oplus \omega_{2}\right)\right)$ is another Hamiltonian $G$-extension of the $N$-action on $X$; here, $G$ acts diagonally on $Z_{1} \times Z_{2}$ and $\iota: X \hookrightarrow Z$ is given by the direct product $\iota(x)=\left(\iota_{1}(x), \iota_{2}(x)\right)$ of the two inclusions $\iota_{j}: X \hookrightarrow Z_{j}, j=1,2$. Our situation can be summarised by the following diagram:

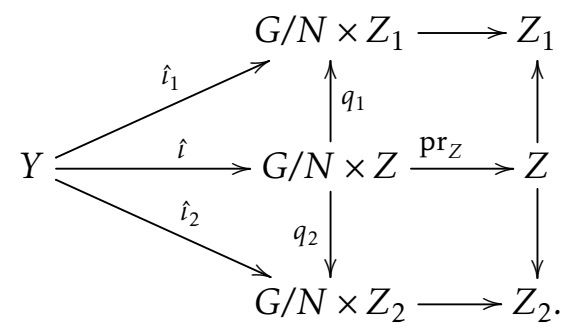

We denote the Kähler form $\omega_{V} \oplus \omega_{j}$ on $G / N \times Z_{j}$ by $\hat{\omega}_{j}$, see Equation (4.2), and note that by assumption the same form $\omega_{V}$ appears in both formulas. Then, it follows from the general construction and from the diagram above that

$$
\omega_{Y, 2}-\omega_{Y, 1}=\hat{l}^{*}\left(q_{2}^{*}\left(\hat{\omega}_{2}\right)-q_{1}^{*}\left(\hat{\omega}_{1}\right)\right)=\hat{\iota}^{*}\left(\operatorname{pr}_{Z}^{*}\left(\omega_{2}-\omega_{1}\right)\right) .
$$

Let $\overline{G / N}$ as defined in Equation (3.4) and let us denote the natural projection $\overline{G / N} \times Z \rightarrow Z$ by $\overline{\mathrm{pr}}_{Z}$. As $Z$ is a Hamiltonian $G$-extension, by Proposition 3.23 there exists a $G$-equivariant holomorphic embedding $\psi: \bar{Y} \hookrightarrow \overline{G / N} \times Z$. It now follows from Equation (4.4) that

$$
\alpha:=\psi^{*}\left(\overline{\operatorname{pr}}_{Z}^{*}\left(\omega_{2}-\omega_{1}\right)\right) \in \mathcal{A}^{1,1}(\bar{Y})
$$

fulfils property (a), as desired.

We still must show that the $K$-invariant 2 -form $\alpha$ is cohomologuous to zero on $\bar{Y}$. For this, we consider the holomorphic fibre bundle $q:=\operatorname{pr}_{\overline{G / N}} \circ \psi: \bar{Y} \hookrightarrow \overline{G / N} \times Z \rightarrow \overline{G / N}$ with typical fibre $X$ and base $\overline{G / N}$. Since $\bar{Y}$ is Kähler, and as by Remark 3.24 the manifold $\overline{G / N}$ is simply-connected, it follows from [Bla56, Théorème II.1.1] that the transgression map $H^{1}(X, \mathbb{R}) \rightarrow H^{2}(\overline{G / N}, \mathbb{R})$ is zero. Consequently, 
the Leray spectral sequence for $q$ degenerates at the $E_{2}$-term by [Bla56, Théorème II.1.2]; see also [Voi07, Theorem 4.15 and Remark 4.16]. Again using simple-connectedness of $\overline{G / N}$, we conclude that

$$
H^{k}(\overline{G / N}, \mathbb{R}) \otimes H^{l}(X, \mathbb{R})=E_{2}^{k, l}=E_{\infty}^{k, l}=\operatorname{Gr}^{k} H^{k+l}(\bar{Y}, \mathbb{R}) \quad \text { and } \quad E_{\infty}^{1,1}=\{0\} .
$$

Computing the corresponding filtration of $H^{2}(\bar{Y}, \mathbb{R})$ and comparing it with the Leray spectral sequence for $\operatorname{pr}_{\overline{G / N}}$ then leads to the following commutative diagram

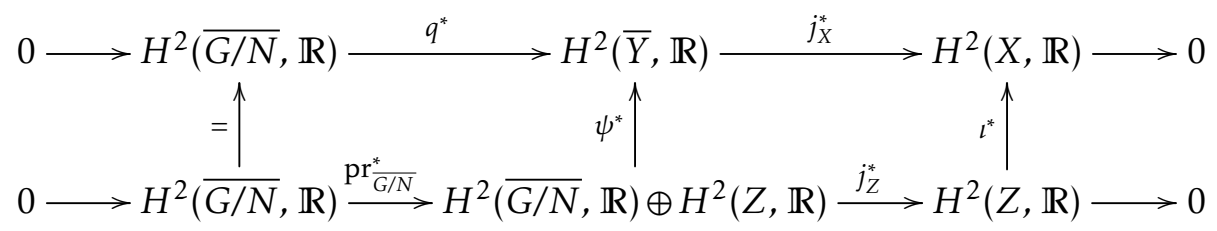

where $j_{X}: X \hookrightarrow \bar{Y}$ is the inclusion as the fibre over $e N \in G / N$, and similarly for $j_{Z}$.

Now, Equation (4.5) says that $[\alpha]=\psi^{*}\left([0] \oplus\left[\omega_{2}-\omega_{1}\right]\right)$. Together with $\left[j_{X}^{*}(\alpha)\right]=\left[\iota_{2}^{*}\left(\omega_{2}\right)\right]-\left[\iota_{1}^{*}\left(\omega_{1}\right)\right]=$ $\left[\omega_{X}\right]-\left[\omega_{X}\right]=0$ this implies that $[\alpha]=0$, as claimed.

Remark 4.5. Note that in contrast to their difference the forms $\omega_{Y, j}$ themselves do not extend to the compactification $\bar{Y}$.

\section{C. Discussion regarding the choice of Kähler metric on $G / N$}

As this is a subtle issue, let us discuss the choice of Kähler forms on $G / N$ made in Section 4.A.a and the fact that we have to fix such a form in some detail. We will provide examples showing that the independence statement of Theorem 4.4 is optimal from many points of view. The problems occuring are closely related to the ones encountered in the algebraic situation when searching for various kinds of "reductive envelopes", cf. [DK07, Sections 5.2 and 5.3].

\section{C.a. The algebraic situation}

Let us compare with Doran-Kirwan's approach in the algebraic situation, see Section 4.A.a: The key point that explains the choice of the trivial line bundle on $G / N$ and that eventually makes the proof of [DK07, Proposition 5.1.9] on the independence of semistability from the choice of the embedding into $G$ work is the following. Given any pair $G$ and $G^{\prime}$ of reductive groups such that $N \subset G \subset G^{\prime}$, the line bundles $L$ on $G \times_{N} X$ and $L^{\prime}$ on $G^{\prime} \times_{N} X$ constructed in [DK07] as above verify $\iota^{*} L^{\prime}=L$ where $\iota: G \times_{N} X \hookrightarrow G^{\prime} \times_{N} X$ is the embedding induced by the inclusion $G \hookrightarrow G^{\prime}$. In the analytic category, such a canonical choice of Kähler metric on $G / N$ does not exist, even among curvature forms in the trivial line bundle. Indeed, every $K$-invariant Kähler metric of the form $\omega=i \partial \bar{\partial} \rho$ with $\rho \in \mathcal{C}^{\infty}(G / N)^{K}$ is the curvature form of a $K$-invariant hermitian metric in the trivial line bundle on $G / N$, cf. [Dem12, Chapter V, (12.6)]. Even if we restrict to metrics of this form, the set of semistable points might change, as the following example shows.

Example 4.6. Let us consider the algebraic and hence meromorphic action of $\mathbb{C} \cong N \subset G=\operatorname{SL}(2, \mathbb{C})$ on $X=\mathbb{P}_{1}$, endowed with the Fubini-Study form $\omega_{F S}$. As Hamiltonian $G$-extension of the $N$-action on $X$ we take $Z=X$. Let $K=\mathrm{SU}(2)$.

According to [Dem12, Lemma 7.10], every $K$-invariant Kähler form on $G / N \cong \mathbb{C}^{2} \backslash\{0\}$ is of the form $i \partial \bar{\partial} \rho(\|z\|)$ where $\rho$ is a smooth function on $\mathbb{R}^{>0}$ such that $\rho \circ \exp$ is strictly increasing and strictly convex. Let $\varphi \in \mathcal{C}^{\infty}\left(\mathbb{R}^{>0}\right)$ be the function defined by $\varphi\left(t^{2}\right)=\rho(t)$. Then the unique moment map for the action of $\mathrm{SU}(2)$ on $\mathbb{C}^{2} \backslash\{0\}$ is given by

$$
z=\left(z_{1}, z_{2}\right) \mapsto \varphi^{\prime}\left(\|z\|^{2}\right)\left(\begin{array}{cc}
\frac{\left|z_{1}\right|^{2}-\left|z_{2}\right|^{2}}{2} & \overline{z_{1}} z_{2} \\
z_{1} \overline{z_{2}} & -\frac{\left|z_{1}\right|^{2}-\left|z_{2}\right|^{2}}{2}
\end{array}\right) .
$$


We now consider the one-parameter family of Kähler forms $\omega_{G / N, c}$ given by $\omega_{G / N, c}:=i \partial \bar{\partial} \rho_{c}(\|z\|)$, where $\rho_{c}(t)=c \log \left(1+t^{2}\right)$ with $c>0$. Following the construction of Section 4.A.a, the induced Kähler form on $Y=G / N \times \mathbb{P}_{1}$ is $\omega_{c}=i \partial \bar{\partial} \rho_{c}(\|z\|) \oplus \omega_{F S}$. Identifying $\mathfrak{s u}(2)^{*}$ with $i \mathfrak{s u}(2)$ via the Killing form, the corresponding moment map $\mu:(G / N) \times \mathbb{P}_{1} \rightarrow i \mathfrak{s u}(2)$ is given by

$$
\mu\left(z,\left[x_{0}: x_{1}\right]\right)=\frac{c}{1+\|z\|^{2}}\left(\begin{array}{cc}
\frac{\left|z_{1}\right|^{2}-\left|z_{2}\right|^{2}}{2 \overline{z_{2}}} & -\overline{z_{1}} z_{2} \\
z_{1} \overline{z_{2}} & -\frac{\left|z_{1}\right|^{2}-\left|z_{2}\right|^{2}}{2}
\end{array}\right)+\frac{1}{\left|x_{0}\right|^{2}+\left|x_{1}\right|^{2}}\left(\begin{array}{cc}
\frac{\left|x_{0}\right|^{2}-\left|x_{1}\right|^{2}}{2} & \overline{x_{0}} x_{1} \\
x_{0} \overline{x_{1}} & -\frac{\left|x_{0}\right|^{2}-\left|x_{1}\right|^{2}}{2}
\end{array}\right) .
$$

A slice for the $\mathrm{SU}(2)$-action on $(G / N) \times \mathbb{P}_{1}$ is given by $S=\{((z, r),[0: 1]) \mid z \in \mathbb{C}, r \geq 0\}$. The point $((z, r),[0: 1]) \in S$ is mapped under $\mu$ to

$$
\frac{c}{1+|z|^{2}+r^{2}}\left(\begin{array}{cc}
\frac{|z|^{2}-r^{2}}{2}-\frac{1}{2} & r \bar{z} \\
r z & -\frac{|z|^{2}-r^{2}}{2}+\frac{1}{2}
\end{array}\right) \text {. }
$$

Consequently, $\mu^{-1}(0)$ is non-empty if and $(c-1)|z|^{2}=1$ for some $z \in \mathbb{C}^{*}$, which is the case if and only if $c>1$. In summary, we see that, depending on $c>0$, the set of semistable points $X_{N}^{s S}$ can be empty or not.

\section{C.b. Proper moment maps}

Notice that Example 4.6 shows that for non-projective $G$-varieties in general the set of GIT-semistable points for the linearisation of the $G$-action in an ample line bundle $L$ and the set of semistable points with respect to a moment map $\mu$ computed using the curvature form of a Hermitian metric in the same line bundle $L$ do not have to coincide. In case the moment map under discussion is proper, the two sets coincide by [Sj95, Theorem 2.18]. Hence, in the above example one could look for Kähler forms leading to proper moment maps that would then give a link to the algebraic theory and establish independence of semistabilty from the choice of the metric.

Looking at formula (4.6) one sees that a moment map of the most general form possible in the given situation is proper on $\mathbb{C}^{2} \backslash\{0\}$ if and only if

$$
\lim _{t \rightarrow 0} \varphi^{\prime}(t) t=\lim _{t \rightarrow \infty} \varphi^{\prime}(t) t=\infty .
$$

Since $t \mapsto \rho\left(e^{t}\right)=\varphi\left(e^{2 t}\right)$ is strictly increasing and strictly convex, we see that $t \mapsto 2 \varphi^{\prime}\left(e^{2 t}\right) e^{2 t}$ is strictly increasing. Hence, $\lim _{t \rightarrow 0} \varphi^{\prime}(t) t=\infty$ is impossible. This proves that there is no proper SU(2)-equivariant moment map on $\mathbb{C}^{2} \backslash\{0\}$. Using compactness of $\mathbb{P}^{1}$, one can use this to conclude that none of the moment maps for the corresponding Kähler forms on $G / N \times \mathbb{P}^{1}$ is proper either, so that proper moment maps just do not exist in the situation at hand.

\section{C.c. Metrics arising from embedding into representations}

As we have seen in Section 2.D, a natural choice in the situation at hand is to consider Kähler metrics on $G / N$ that are obtained by embedding this homogeneous space as an orbit in a $G$-representation space, and this is also the choice made in the construction presented in Section 4.A.a. We will see in Section 5 below that this leads to a number of desirable properties. However, also a restriction to this class of metrics does not lead to a common notion of semistability, as the following example shows.

Example 4.7. We continue the discussion at the end of Example 2.9 and consider $G=\operatorname{SL}(3, \mathbb{C})$ and $N=G_{v}$ the unipotent radical of a certain Borel subgroup of $G$. If we equip $G / N$ with the restriction of the flat Kähler metric for which the image of the moment map $\mu_{V}$ has complement with non-empty interior, and if we take $X=X_{\alpha}$ to be the $G$-flag manifold corresponding to the coadjoint orbit $\operatorname{Ad}^{*}(K) \bullet \alpha$ through a point $\alpha \in \mathrm{k}_{\text {reg }}^{*}$ such that $-\alpha$ does not lie in the image of $\mu_{V}$, then the set of semistable points for the $G$-action on $G \times{ }_{N} X$ is empty. On the other hand, as $N$ is a Grosshans subgroup of $G$, there exists a $G$-module $V^{\prime}$ inducing a moment map $\mu_{V^{\prime}}$ on $G / N$ whose image is a $K$-invariant dense open subset of $\mathrm{k}^{*}$, see Lemma 2.8. Without loss of generality, we may assume that the point $\alpha \in \mathrm{k}_{\text {reg }}^{*}$ chosen above fulfils $-\alpha \in \mu_{V^{\prime}}(G / N)$. Then, for the Kähler metric on $G \times_{N} X$ induced by the second embedding the set of semistable points is not empty. 


\section{C.d. Unipotent radicals of parabolic subgroups}

The next example shows that even in the case that we are able to embed $N$ as the unipotent radical of parabolic subgroups of two different semisimple groups $G_{1}$ and $G_{2}$ and hence, as explained in Remark 2.10, for each of the two embeddings there exists a very natural choice of a Kähler form on $G_{j} / N$, we cannot expect $X_{N}^{s S}$ to be independent of the group $G_{j}$.

Example 4.8. Let us consider the action of $N=\mathbb{C}^{2}$ on $X=\mathbb{P}_{2}$ given by the embedding

$$
N \hookrightarrow G_{1}=\mathrm{SL}(3, \mathbb{C})=\mathrm{SU}(3)^{\mathbb{C}}=K_{1}^{\mathbb{C}}, \quad(t, s) \mapsto\left(\begin{array}{ccc}
1 & 0 & t \\
0 & 1 & s \\
0 & 0 & 1
\end{array}\right) .
$$

Taking the obvious Hamiltonian $G_{1}$-extension $Z_{1}=X$, we have $G_{1} \times_{N} X=G_{1} \times_{N} Z_{1}=\left(G_{1} / N\right) \times \mathbb{P}_{2}$ with moment map $\mu=\mu_{V}+\mu_{\mathbb{P}_{2}}:\left(G_{1} / N\right) \times \mathbb{P}_{2} \rightarrow k_{1}^{*}=\mathfrak{s u}(3)^{*}$. Since $N$ is embedded as the unipotent radical of a parabolic subgroup $P$ of $G_{1}$, we may consider the canonical affine completion $\bar{G}_{1} / N^{\text {a }}$ and equip it with the canonical Kähler form that is described in the paragraph before Remark 3.4 in [Kir11], cf. Remark 2.10.

The behaviour of the corresponding moment map $\mu_{V}$ on $\bar{G}_{1} / N^{a}$ is best understood in terms of its description as the universal $K^{(P)}$-imploded cross-section $\left(T^{*} K\right)_{\text {impl }}^{K, K^{(P)}}$, see [Kirll, Definition 3.11]. According to the discussion following Remark 3.13 in [Kirl1], the $G_{1}$-orbits in ${\overline{G_{1}} / N^{a}}^{\text {a }}$ correspond to the strata

$$
\left(K_{1} \times \operatorname{Ad}^{*}\left(K_{1}^{(P)}\right) \cdot \sigma\right) / \approx_{K_{1}^{(P)}},
$$

where $\sigma$ runs through the open faces of $\left(\mathrm{t}_{1}^{*}\right)_{+}$. In particular, the open orbit $G_{1} / N$ is associated with the interior $\operatorname{int}\left(\mathrm{t}_{1}^{*}\right)_{+}$of $\left(\mathrm{t}_{1}^{*}\right)_{+}$. The description of the moment map $\mu_{V}$ given in [Kirll, Theorem 3.12] now implies that $\mu_{V}\left(G_{1} / N\right)$ is contained in $\left(k_{1}\right)_{\text {reg }}^{*}=\operatorname{Ad}^{*}\left(K_{1}\right) \cdot \operatorname{int}\left(\mathrm{t}_{1}^{*}\right)_{+}$. Since $\mu_{\mathbb{P}_{2}}\left(\mathbb{P}_{2}\right)$ does not intersect the interior of $\left(\mathrm{t}_{1}^{*}\right)_{+}$, the zero fibre of $\mu$ is empty, hence $X_{N}^{s S}=\emptyset$.

Now, let us consider the second embedding

$$
N \hookrightarrow G_{2}=\mathrm{SL}(2, \mathbb{C}) \times \mathrm{SL}(2, \mathbb{C})=(\mathrm{SU}(2) \times \mathrm{SU}(2))^{\mathbb{C}}=K_{2}^{\mathbb{C}}, \quad(t, s) \mapsto\left(\left(\begin{array}{ll}
1 & t \\
0 & 1
\end{array}\right),\left(\begin{array}{ll}
1 & s \\
0 & 1
\end{array}\right)\right) .
$$

Here, $N$ is embedded as the unipotent radical of a Borel subgroup of $G_{2}$, and thus in particular again a Grosshans subgroup of $G_{2}$. As $G_{2}$-extension of the $N$-action on $X=\mathbb{P}_{2}$ we choose the embedding

$$
\iota: \mathbb{P}_{2} \hookrightarrow \mathbb{P}_{3}, \quad \iota\left(\left[z_{0}: z_{1}: z_{2}\right]\right)=\left[z_{0}: z_{2}: z_{1}: z_{2}\right],
$$

which is $N$-equivariant for the $N$-action on $\mathbb{P}_{3}$ given by

$$
N \hookrightarrow G_{2} \hookrightarrow \operatorname{SL}(4, \mathbb{C}), \quad(t, s) \mapsto\left(\begin{array}{llll}
1 & t & 0 & 0 \\
0 & 1 & 0 & 0 \\
0 & 0 & 1 & s \\
0 & 0 & 0 & 1
\end{array}\right)
$$

A moment map $\mu_{\mathbb{P}_{3}}: \mathbb{P}_{3} \rightarrow \mathfrak{s u}(2) \oplus \mathfrak{s u}(2)$ for the $K_{2}$-action on $\mathbb{P}_{3}$ with respect to the Fubini-Study metric is given by the explicit formula

$$
\mu_{\mathbb{P}_{3}}\left(\left[z_{0}: z_{1}: z_{2}: z_{3}\right]\right)=\frac{1}{\left|z_{0}\right|^{2}+\cdots+\left|z_{3}\right|^{2}}\left[\left(\begin{array}{cc}
\frac{\left|z_{0}\right|^{2}-\left|z_{1}\right|^{2}}{2} & \overline{z_{0}} z_{1} \\
z_{0} \overline{z_{1}} & -\frac{\left|z_{0}\right|^{2}-\left|z_{1}\right|^{2}}{2}
\end{array}\right) \oplus\left(\begin{array}{cc}
\frac{\left|z_{2}\right|^{2}-\left|z_{3}\right|^{2}}{2} & \overline{z_{2}} z_{3} \\
z_{2} \overline{z_{3}} & -\frac{\left|z_{2}\right|^{2}-\left|z_{3}\right|^{2}}{2}
\end{array}\right)\right],
$$

see Example 4.6. In order to determine the semistable locus $Y_{G_{2}}^{s s}\left(\mu_{Y}\right)$ in $Y=G_{2} \times_{N} X$ we consider the closed embedding

$$
Y=G_{2} \times_{N} X \hookrightarrow G_{2} \times_{N} Z \cong G_{2} / N \times \mathbb{P}_{3}
$$


and the moment map $\mu_{Y}=\left.\left(\mu_{V}+\mu_{\mathbb{P}_{3}}\right)\right|_{Y}$. The canonical affine closure of

$$
G_{2} / N \cong\left(\mathbb{C}^{2} \backslash\{0\}\right) \times\left(\mathbb{C}^{2} \backslash\{0\}\right)
$$

is $V=\mathbb{C}^{2} \oplus \mathbb{C}^{2}=\mathbb{C}^{4}$, which we equip with the Hermitian structure $\frac{1}{2}\langle\cdot, \cdot\rangle_{s t}$, where $\langle\cdot, \cdot\rangle_{s t}$ is the standard Hermitian product of $\mathbb{C}^{4}$. A direct calculation using the formulae given in Example 4.6 and the explicit expression for $\mu_{\mathbb{P}_{3}}$ given above yields

$$
\mu_{Y}(e N,[0: 0: 1])=\mu_{V}((1,0),(1,0))+\mu_{\mathbb{P}_{3}}([0: 1: 0: 1])=0 .
$$

Hence, we have $X_{N}^{s s} \neq \emptyset$.

\section{D. Semistable points induced by affine completions of $G / N$}

There is a further way to define $N$-semistable points in $X$, less directly linked to the intrinsic geometry of $G / N$ and $X$. Instead of discussing the diagonal $G$-action on $G / N \times Z$ let us consider an affine completion $\overline{G / N}^{\mathrm{a}}$ and consider the diagonal $G$-action on $\overline{G / N}^{\mathrm{a}} \times Z$. Let $\bar{l}: X \hookrightarrow \overline{G / N}^{\mathrm{a}} \times Z$ be the $N$ equivariant embedding and define $X_{N}^{\overline{s s}}\left[\omega_{X}\right]:=\bar{\imath}^{-1}\left(\left(\overline{G / N}^{\mathrm{a}} \times Z\right)_{G}^{s S}\left[\omega_{V}+\omega_{Z}\right]\right)$. Then, $X_{N}^{\overline{s s}}\left[\omega_{X}\right]$ is an open $N$-invariant subset which contains but in general is strictly bigger than $X_{N}^{s S}\left[\omega_{X}\right]$. Analogously, we define $X_{N}^{\bar{s}}$ as $\bar{l}^{-1}\left(\left(\overline{G / N}^{\mathrm{a}} \times Z\right)_{G}^{s}\left[\omega_{V}+\omega_{Z}\right]\right)$.

Lemma 4.9. Let $\left(X, \omega_{X}\right)$ be a compact Hamiltonian $N$-manifold with a Hamiltonian G-extension $\left(Z, \omega_{Z}\right)$. If $N$ is a Grosshans subgroup of $G$, i.e., if $\mathbb{C}[G]^{N}$ is finitely generated, then for the canonical affine completion $\operatorname{Spec} \mathbb{C}[G]^{N}$ of $G / N$ the set $X_{N}^{\overline{s s}}$ is non-empty.

Proof. We already noticed in Section 2.D that under the Grosshans assumption the corresponding moment map $\mu_{V}: \operatorname{Spec} \mathbb{C}[G]^{N} \rightarrow \mathfrak{k}^{*}$ is surjective. Every moment map $\mu=\mu_{V}+\mu_{Z}: \operatorname{Spec} \mathbb{C}[G]^{N} \times Z \rightarrow \mathfrak{k}^{*}$ thus has non-empty zero fibre.

\section{E. Algebraic actions on projective manifolds}

In this section, we study the following situation: let $X$ be a projective manifold and $N$ a unipotent group acting linearly on $X$ in the sense that there exists a finite-dimensional $N$-representation $W$ such that the corresponding homomorphism $N \rightarrow \mathrm{GL}(W)$ embeds $N$ into a semisimple subgroup $G$ of $\operatorname{SL}(W)$, and an $N$-equivariant embedding $\iota: X \hookrightarrow \mathbb{P}(W)$. We will compare the moment map approach presented in earlier sections with the Geometric Invariant Theory approach of Doran-Kirwan [DK07].

Consider the (very ample) line bundle $L_{X}:=l^{*}\left(\mathscr{O}_{\mathbb{P}(W)}(1)\right)$ on $X$, which is $N$-linearised by construction. Let $\langle\cdot, \cdot\rangle$ be a Hermitian inner product on $W$ and set $K:=\operatorname{SU}(W,\langle\cdot, \cdot\rangle) \cap G$, so that $G=K^{\mathbb{C}}$. Endow $\mathbb{P}(W)$ and hence $X$ with the corresponding Fubini-Study Kähler form $\omega_{F S}$ and its restriction $\omega_{X}:=\iota^{*}\left(\omega_{F S}\right)$, respectively, so that $\left[\omega_{X}\right]=c_{1}\left(L_{X}\right) \in H^{2}(X, \mathbb{R})$. Note that $\mathbb{P}(W)$ is a Hamiltonian $G$-extension of $X$. Next, as suggested by the construction of semistable points with respect to $\omega_{X}$, we look at

$$
\bar{\imath}: X \hookrightarrow Y=G \times_{N} X \hookrightarrow G \times_{N} \mathbb{P}(W) \cong G / N \times \mathbb{P}(W) \hookrightarrow \overline{G / N}^{\mathrm{a}} \times \mathbb{P}(W),
$$

cf. Section 4.D, and additionally at the $G$-linearised ample line bundle $L:=\mathscr{O}_{\overline{G / N}^{a}} \otimes \mathscr{O}_{\mathbb{P}(W)}(1)$. In this situation, we define

$$
X_{N}^{\bar{s}}\left(L_{X}\right):=\bar{l}^{-1}\left(\left(\overline{G / N}^{\mathrm{a}} \times \mathbb{P}(W)\right)_{G}^{s}(L)\right)
$$

to be the pre-image of the GIT-stable points for the $G$-action on $\overline{G / N}^{\mathrm{a}} \times \mathbb{P}(W)$ and the given linearisation, which is unique as $G$ is semisimple. The main comparison result regarding moment-map-semistability and GIT-semistability can now be formulated as follows:

Proposition 4.10. In the above situation, assume additionally that $\overline{G / N}^{\mathrm{a}}$ is normal. Then, we have

$$
X_{N}^{s s}\left[\omega_{X}\right]=X_{N}^{\bar{s}}\left(L_{X}\right)
$$


Proof. The inner product $\langle\cdot, \cdot\rangle$ induces a $K$-invariant Hermitian metric on $\mathscr{O}_{\mathbb{P}(W)}(1)$ such that $\frac{i}{2 \pi} \times$ the curvature is $\omega_{F S}$. Using a $K$-invariant Hermitian metric on the trivial line bundle over $V \supset \overline{G / N}^{\text {a with }}$ $\frac{i}{2 \pi} \times$ curvature equal to $\omega_{V}$, we get a $K$-invariant Hermitian metric $h$ on $L \rightarrow \overline{G / N}^{\mathrm{a}} \times \mathbb{P}(W)$ with $\frac{i}{2 \pi} \times$ curvature equal to $\omega_{V}+\omega_{F S}$. We are hence in the general situation of $[\mathrm{Sj} 95 \text {, Section } 2.2]^{5}$, with the exception that $\overline{G / N}^{\mathrm{a}} \times \mathbb{P}(W)$ is normal and not smooth, which does not affect Sjamaar's arguments ${ }^{6}$. In particular, the compact complex space $\left(\overline{G / N}^{\mathrm{a}} \times \mathbb{P}(W)\right)_{G}^{S S}\left(\mu \overline{G / N}^{\mathrm{a}} \times \mathbb{P}(W) / / G\right.$ is projective algebraic by Grauert's version of the Kodaira Embedding Theorem, see [Sj95, Theorem 2.17]. Moreover, we claim that

$$
\left(\overline{G / N}^{\mathrm{a}} \times \mathbb{P}(W)\right)_{G}^{s S}\left(\mu_{\overline{G / N}}^{\mathrm{a}} \times \mathbb{P}(W)=\left(\overline{G / N}^{\mathrm{a}} \times \mathbb{P}(W)\right)_{G}^{s s}(L) .\right.
$$

In order to prove this, as the moment map $\mu_{\overline{G / N}} \mathrm{a} \times \mathbb{P}(W)$ is proper we can follow the general line of argumentation presented in [Sj95, proof of Theorem 2.18]: Since the possibly singular variety $\overline{G / N}^{\mathrm{a}} \times \mathbb{P}(W)$ is contained in $V \times \mathbb{P}(W)$, and since all differential geometric and symplectic objects are obtained by restriction, the computations regarding the relation between the norms of sections and (the norm square of) the moment map given in the first paragraph of loc. cit. continue to hold, so that for any $\mu$-semistable $p \in \overline{G / N}^{\mathrm{a}} \times \mathbb{P}(W)$ and any $G$-invariant section $s$ of $L$ over $\left(\overline{G / N}^{\mathrm{a}} \times \mathbb{P}(W)\right)_{G}^{s S}\left(\mu \overline{G / N}^{\mathrm{a}} \times \mathbb{P}(W)\right.$, the restriction of the function $h(s, s)$ to the closure of $G \cdot p$ inside $\left(\overline{G / N}^{\mathrm{a}} \times \mathbb{P}(W)\right)_{G}^{s S}\left(\mu \overline{G / N}^{\mathrm{a}} \times \mathbb{P}(W)\right.$ takes on its maximum at the limit $F_{\infty}(p)$ under the gradient flow of $-\left\|\mu \overline{G / N}^{a} \times \mathbb{P}(W)\right\|^{2}$, from which we conclude that $s$ is bounded on $\left(\overline{G / N}^{\mathrm{a}} \times \mathbb{P}(W)\right)_{G}^{s S}\left(\mu \overline{G / N}^{\mathrm{a}} \times \mathbb{P}(W)\right.$. Furthermore, an application of [Gre10a, Proposition 7.6] shows that the set of $\mu$-semistable points is Zariski-open. Since in addition $\overline{G / N}^{\mathrm{a}} \times \mathbb{P}(W)$ is normal, it therefore follows from Riemann's Extension Theorem ${ }^{7}$ that $s$ extends to a $G$-invariant section over the whole of $\overline{G / N}^{\mathrm{a}} \times \mathbb{P}(W)$. The arguments for the two implications "algebraically semistable implies analytically semistable" and "analytically semistable implies algebraically semistable" can now be used without changes, proving (4.8).

The analogous equality for stable points follows from the fact that on both sides, these are the ones for which the corresponding fibre of the quotient map consists of a single (closed) orbit. Intersecting with $\bar{\imath}(X)$ yields $X_{N}^{\bar{s}}\left[\omega_{X}\right]=X_{N}^{\bar{s}}\left(L_{X}\right)$, from which we conclude using Corollary 5.2 proven in Section 5.A below.

Remark 4.11. (Comparison of semistable points) In the given situation, Doran and Kirwan in [DK07, Definition 5.1.6] define the set of GIT-semistable points to be

$$
X_{N}^{s s}\left(L_{X}\right):=X \cap Y_{G}^{s s}\left(\hat{\imath}^{*} \mathscr{O}_{G / N} \otimes \mathscr{O}_{\mathbb{P}(W)}(1)\right),
$$

where $\hat{\imath}$ is given by (4.1). In general, this set will not coincide with $X_{N}^{s S}\left[\omega_{X}\right]$, as the following argument shows. Assume we had $X_{N}^{s S}\left[\omega_{X}\right]=X_{N}^{s s}\left(L_{X}\right)$. Since the latter set only depends on the $N$-action on $X$ and its lift to the $N$-linearised line bundle $L_{X}$, see [DK07, Proposition 5.1.9], the same would be true for $X_{N}^{s S}\left[\omega_{X}\right]$. In particular, $X_{N}^{s S}\left[\omega_{X}\right]$ would be independent of the chosen embedding $N \hookrightarrow G$ and of the chosen embedding $G / N \hookrightarrow V$ with (normal) affinisation $\overline{G / N}^{\text {a }}$. This however would stand in contradiction to Example 4.8.

In this direction, Equality (4.7) gives the inclusion $X_{N}^{s S}\left[\omega_{X}\right] \subset X_{N}^{S S}\left(L_{X}\right)$, which in general is strict, as the gradient flow of the norm square of the moment map of a GIT-semistable point in $\hat{\imath}(Y)$ might converge to a point (in the zero fibre of the moment map) in the boundary of $\hat{\imath}(Y)$ in $\overline{G / N}^{\mathrm{a}} \times \mathbb{P}(W)$.

\section{Properties of quotients by unipotent groups}

We establish the existence of a compactifiable geometric quotient of the set of semistable points by the $N$-action that extends to a meromorphic map from $X$ to the compactification and carries a natural Kähler

\footnotetext{
$5 \uparrow$ As $G$ is semisimple, the moment map computed there has to coincide with $\mu \overline{G / N}^{\mathrm{a}} \times \mathbb{P}(W)$.

$6 \uparrow$ See also [HaH99] for the result attributed to Roberts. Regarding [Sj95, Lemma 2.16], see also [HHL94].

$7 \uparrow$ In Sjamaar's setup the application of Riemann's Extension Theorem is not justified, since at this point the complement of the set of $\mu$-semistable points is not known to be small enough; e.g., it could contain interior points (in the Euclidean topology).
} 
form obtained by symplectic reduction. We will use the notation established in Section 4.A.a.

\section{A. Existence of geometric quotients}

As in the reductive case, sets of semistable points admit quotients, which in the unipotent case are automatically geometric, since unipotent groups cannot have properly semistable orbits by the following

Lemma 5.1. Let $\left(X, \omega_{X}\right)$ be a Hamiltonian $N$-manifold. Then every $N$-orbit in $X_{N}^{s s}\left[\omega_{X}\right]$ is closed in $X_{N}^{s s}\left[\omega_{X}\right]$, i.e., we have $X_{N}^{s s}\left[\omega_{X}\right]=X_{N}^{s}\left[\omega_{X}\right]$.

Proof. Consider the analytic Hilbert quotient $\pi_{G}: Y_{G}^{s s}\left[\omega_{Y}\right] \rightarrow Y_{G}^{s s}\left[\omega_{Y}\right] / / G$. The fibre $\pi_{G}^{-1}\left(\pi_{G}(x)\right)$ is an affine $G$-variety, see [HH99, Proposition 3.3.7]. It hence follows from a classical result that every $N$-orbit is closed in $\pi_{G}^{-1}\left(\pi_{G}(x)\right)$ and hence in $Y_{G}^{s s}\left[\omega_{X}\right]$. The claim follows.

Corollary 5.2. In the situation of Section 4.D, we have $X_{N}^{s s}\left[\omega_{X}\right]=X_{N}^{\bar{s}}\left[\omega_{X}\right]$.

Proof. It follows from Lemma 5.1 that every orbit in $Y_{G}^{s s}\left[\omega_{Y}\right]$ is closed in $Y_{G}^{s s}\left[\omega_{Y}\right]$. If $\Phi: Y \hookrightarrow \overline{G / N}^{\mathrm{a}} \times Z$ is the natural inclusion, we hence have

$$
Y^{s s}\left[\omega_{Y}\right]=\left\{y \in Y \mid G \cdot y \cap \mu_{Y}^{-1}(0) \neq \emptyset\right\}=\left\{y \in Y \mid G \cdot \Phi(y) \cap \mu_{\overline{G / N} \times Z}^{-1}(0) \neq \emptyset\right\} .
$$

As $\Phi$ restricted to $X \subset Y$ coincides with $\bar{l}$, the claim follows.

Theorem 5.3. Let $\left(X, \omega_{X}\right)$ be a compact Hamiltonian $N$-manifold. Then, the set $X_{N}^{s S}\left[\omega_{X}\right]$ of semistable points admits a geometric quotient $\pi: X_{N}^{S S}\left[\omega_{X}\right] \rightarrow X_{N}^{S S}\left[\omega_{X}\right] / N$ by the $N$-action. In fact, $\pi$ is a principal $N$-fibre bundle and $X_{N}^{s S}\left[\omega_{X}\right] / N=: Q$ is smooth.

Proof. By the quotient theory for Hamiltonian actions of reductive groups, see Theorem 2.6, the set of $G$-semistable points $Y_{G}^{s S}\left[\omega_{Y}\right]=G \cdot X_{N}^{s s}\left[\omega_{X}\right]$ admits an analytic Hilbert quotient by the $G$-action. Moreover, by Lemma 5.1, every $G$-orbit in $Y_{G}^{s s}\left[\omega_{Y}\right]$ is closed there, hence the quotient $Y_{G}^{s s}\left[\omega_{Y}\right] \rightarrow Y_{G}^{s s}\left[\omega_{Y}\right] / / G$ is in fact geometric. By construction of the twisted product, the restriction to $X_{N}^{s S}\left[\omega_{X}\right] \subset Y_{G}^{s S}\left[\omega_{Y}\right]$ yields the desired geometric quotient $\pi$.

For every $x \in X_{N}^{s S}\left[\omega_{X}\right]$, the $G$-orbit is closed, hence the isotropy subgroup $G_{x}$ is reductive. On the other hand, as $x \in X$, the isotropy is contained in $N$, and hence unipotent. It follows that $G_{x}=N_{x}=\{e\}$, and hence that $\pi$ is a principal $N$-fibre bundle.

\section{B. Compactifications of the quotient}

We will establish the existence of natural compactifications of the quotient $X_{N}^{s S}\left[\omega_{X}\right] / N$, which we assume to be non-empty in this section.

Recall that the fundamental construction of Section 4.A.a involves the choice of an embedding of $G / N$ into a Hermitian $K$-representation $V$ as a $G$-orbit $G \cdot v$, see Equation (4.2). This leads to an affine completion $\overline{G / N}^{\mathrm{a}}:=\overline{G \cdot v}$ of $G / N$ to which both the Kähler form and the moment map extend. Consider the composition $\Phi: Y \hookrightarrow \overline{G / N}^{\mathrm{a}} \times Z$ of the open embedding $G / N \times Z \hookrightarrow \overline{G / N}^{\mathrm{a}} \times Z$ with the embedding (4.1) used in the main construction.

Proposition 5.4. The inclusion $Y=G \times_{N} X \stackrel{\Phi}{\hookrightarrow} \overline{G / N}^{\mathrm{a}} \times Z$ induces an open embedding

$$
\phi: X_{N}^{s S}\left[\omega_{X}\right] / N \hookrightarrow \bar{Q}
$$

of $X_{N}^{s s}\left[\omega_{X}\right] / N=Q$ into a compact complex space $\bar{Q}$ such that $\bar{Q} \backslash \phi(Q)$ is analytic and nowhere dense. 
Proof. We claim that $\Phi(Y)$ is Zariski-open in its closure. For this, we first look at the compactification $V \times Z \hookrightarrow \mathbb{P}(V \oplus \mathbb{C}) \times Z$; by slight abuse of notation, the composition of $\Phi$ with this embedding will also be denoted by $\Phi$. Since the $G$-action on the compact Kähler manifold $Z$ is Hamiltonian, it is meromorphic, see Remark 2.2. As in addition the $G$-action on $V$ is algebraic, the $G$-action on the compact Kähler manifold $\mathbb{P}(V \oplus \mathbb{C}) \times Z$ is meromorphic. Secondly, we notice that $\Phi(Y)=G \bullet(\{e U\} \times \iota(X))$, where $\iota: X \hookrightarrow Z$ is the extension map; i.e., $\Phi(Y)$ is the $G$-sweep of a compact complex submanifold of $\mathbb{P}(V \oplus \mathbb{C}) \times Z$. It therefore follows from [Fuj78, Lemma 2.4(1)] that $\Phi(Y)$ is Zariski-open in its closure inside $\mathbb{P}(V \oplus \mathbb{C}) \times Z$, and hence it is Zariski-open in its closure inside $\overline{G / N}^{\mathrm{a}} \times \mathrm{Z}$. We denote this closure by $\bar{Y}$.

By Theorem 2.7 the moment map $\mu_{V}$ is proper on $\overline{G / N}^{\mathrm{a}}$. It follows that the moment map for the action of $K$ on $\overline{G / N}^{\mathrm{a}} \times Z$ and hence the restriction $\mu_{\bar{Y}}: \bar{Y} \rightarrow \mathfrak{k}^{*}$ of this moment map to the analytic subset $\bar{Y} \subset \overline{G / N}^{\mathrm{a}} \times Z$ is likewise proper. Recalling the construction of the Kähler form $\omega_{Y}$ and the associated moment map $\mu_{Y}$, we can summarise the situation in the following commutative diagram:

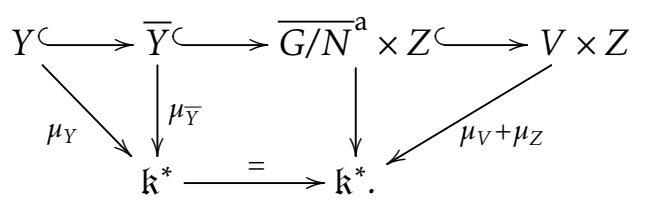

Here, in the first line, the first inclusion is open and the other two inclusions are closed.

Since $\mu_{\bar{Y}}$ is proper, its zero fibre is compact, and hence the associated analytic Hilbert quotient $\bar{Y}_{G}^{s s}\left(\mu_{\bar{Y}}\right) / / G \simeq \mu_{\bar{Y}}^{-1}(0) / K$ is a compact complex space, which in fact comes with a natural closed embedding into the (non-compact) analytic Hilbert quotient $(V \times Z)_{G}^{s s}\left(\mu_{V}+\mu_{Z}\right) / / G$. As the inclusion $Y \hookrightarrow \bar{Y}$ has Zariski-open image, and as every $G$-orbit in $Y_{G}^{s s}\left(\mu_{Y}\right)=Y_{G}^{s s}\left[\omega_{Y}\right]$ is closed by the argument in the proof of Theorem 5.3, the inclusion $Y_{G}^{s s}\left[\omega_{Y}\right] \hookrightarrow \bar{Y}_{G}^{s s}\left(\mu_{\bar{Y}}\right)$ is Zariski-open and saturated with respect to the quotient map $\bar{\pi}: \bar{Y}_{G}^{s s}\left(\mu_{\bar{Y}}\right) \rightarrow \bar{Y}_{G}^{s s}\left(\mu_{\bar{Y}}\right) / / G$. It therefore induces the desired Zariski-open embedding

$$
\phi: Q=X_{N}^{s s}\left[\omega_{X}\right] / N \cong Y_{G}^{s s}\left[\omega_{Y}\right] / G \hookrightarrow \bar{Y}_{G}^{s s}\left(\mu_{\bar{Y}}\right) / / G=: \bar{Q}
$$

into the compact complex space $\bar{Q}$.

\section{C. Zariski-openness of semistable points and meromorphic extension of the quotient map}

While there is no general result for analyticity of the complement of the set of semistable points in a Hamiltonian G-manifold with non-proper moment map, in our setup this can be shown by hand.

Theorem 5.5. Let $\left(X, \omega_{X}\right)$ be a compact Hamiltonian $N$-manifold. Then, the set $X_{N}^{s s}\left[\omega_{X}\right]$ of semistable points is Zariski-open in X. Moreover, the quotient map $\pi: X_{N}^{s S}\left[\omega_{X}\right] \rightarrow X_{N}^{S S}\left[\omega_{X}\right] / N$ extends to a meromorphic map ${ }^{8}$ $\pi: X \rightarrow \bar{Q}$ to the compact complex space $\bar{Q}$ constructed in the proof of Proposition 5.4.

Proof. By part (1) of Theorem 2.6, $X_{N}^{s s}\left[\omega_{X}\right]$ is open in the Euclidean topology. Let $\pi_{F}: X \rightarrow Q_{F}$ be a Fujiki quotient of $X$ by the $N$-action, whose existence is guaranteed by Proposition 1.4, and let $\Gamma \subset X \times Q_{F}$ be the graph. In particular, $\Gamma$ is an $N$-invariant, irreducible, compact analytic subset of $X \times Q_{F}$, where $N$ acts only on the first factor. Embedding $X \times Q_{F}$ into $Y \times Q_{F}$ and further into $\mathbb{P}(V \oplus \mathbb{C}) \times Z \times Q_{F}$ as in the proof of Proposition 5.4 we can interpret $\Gamma$ as an $N$-invariant subvariety in $\mathbb{P}(V \oplus \mathbb{C}) \times Z \times Q_{F}$. Using again that the $G$-action on the latter space is meromorphic, we conclude that $\hat{\Gamma}:=\overline{G \cdot \Gamma}$ is Zariski-open in its closure in $\bar{Y} \times Q_{F}$, and in particular irreducible. On $Y \subset \bar{Y}$ it is the graph of the $G$-invariant extension of the $N$-invariant meromorphic map $\pi_{F}$ from $X$ to $Y=G \times_{N} X$. It follows that $\hat{\Gamma}$ is the graph of a $G$-invariant

$8 \uparrow$ The reader is referred to [Whi72, Chapter 6, Section 3] for an in depth discussion of meromorphic mappings between complex spaces. 
meromorphic map from $\bar{Y}$ to $Q_{F}$, which we will call $\hat{\pi}_{F}$. The graph of the restriction of $\hat{\pi}_{F}$ to $\bar{Y}_{G}^{s S}\left(\mu_{\bar{Y}}\right)$ is equal to $\hat{\Gamma}^{\circ}:=\hat{\Gamma} \cap\left(\bar{Y}_{G}^{S S}\left(\mu_{\bar{Y}}\right) \times Q_{F}\right)$. Now, $\bar{Y}_{G}^{S S}\left(\mu_{\bar{Y}}\right) \times Q_{F}$ admits an analytic Hilbert quotient by the $G$-action, namely $\Pi=\bar{\pi} \times \operatorname{id}_{Q_{F}}: \bar{Y}_{G}^{s s}\left(\mu_{\bar{Y}}\right) \times Q_{F} \rightarrow \bar{Q} \times Q_{F}$. As $\hat{\Gamma}^{\circ}$ is a $G$-invariant, irreducible analytic subset of $\bar{Y}_{G}^{s S}\left(\mu_{\bar{Y}}\right) \times Q_{F}$, its image $\hat{\Gamma}_{\text {red }}^{\circ}=\Pi\left(\hat{\Gamma}^{\circ}\right)$ is an irreducible analytic subset of $\bar{Q} \times Q_{F}$ by the basic properties of analytic Hilbert quotients listed in Section 2.C.

On the one hand, as orbits through points in $Y_{G}^{s S}\left(\mu_{Y}\right) \subset \bar{Y}_{G}^{S S}\left(\mu_{\bar{Y}}\right)$ are closed, $\hat{\Gamma}_{\text {red }}^{\circ}$ defines a meromorphic map $\pi_{F \text {,red }}$ from $\bar{Q}$ to $Q_{F}$, cf. the argument given in the proof of [Grel0a, Proposition 4.5]. On the other hand, consider the open subset $U:=U_{F} \cap X_{N}^{s S}\left[\omega_{X}\right]$, cf. Proposition 1.4. As this set is $N$-invariant, and since both $\left.\pi_{F}\right|_{U}$ and $\left.\pi\right|_{U}$ are geometric quotients for the $N$-action on $U$ by Proposition 1.4 and Theorem 5.3, respectively, the respective images $\pi_{F}(U) \subset Q_{F}$ and $\pi(U) \subset Q \subset \bar{Q}$ are biholomorphic via $\pi_{F \text {,red. It }}$ follows that $\pi_{F, \text { red }}: \bar{Q} \rightarrow Q_{F}$ is bimeromorphic. From this, we conclude that $\left(\pi_{F, \text { red }}\right)^{-1} \circ \pi_{F}: X \rightarrow \bar{Q}$ is a meromorphic extension of $\pi$ and that there are Zariski-open, dense subsets $\bar{\Omega} \subset \bar{Q}$ and $\Omega_{F} \subset Q_{F}$ that are biholomorphic via $\pi_{F \text {,red }}$. By shrinking $U_{F}$ if necessary, we may assume that $\Omega_{F}=\pi_{F}\left(U_{F}\right)$ and $\bar{\Omega} \subset Q \subset \bar{Q}$. The situation is hence summarised by the following commutative diagram

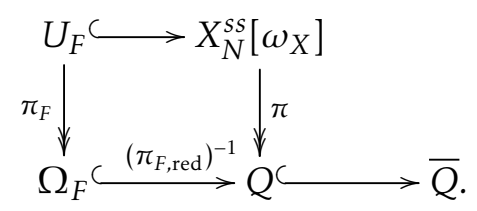

In particular, the Zariski-open subset $U_{F}$ is contained in $X_{N}^{s s}\left[\omega_{X}\right]$. Since $X$ is compact, using a Noetherian induction argument applied to the analytic subset $X^{\prime}:=X \backslash U_{F}$ we conclude that $X_{N}^{s S}\left[\omega_{X}\right]$ and hence $X \backslash X_{N}^{s S}\left[\omega_{X}\right]$ is constructible in the Zariski-topology of $X$. As we know from the start that $X \backslash X_{N}^{s S}\left[\omega_{X}\right]$ is closed in the Euclidean topology of $X$, the claim follows.

\section{D. Reduced Kähler structure on the quotient}

We will show that using a symplectic reduction procedure the quotient $X_{N}^{s s}\left[\omega_{X}\right] / N$ can be endowed with a Kähler form naturally induced from $\omega_{X}$. This form extends to the compactification $\bar{Q}$ and its class pulls back under $\pi$ to the class of $\left[\omega_{X}\right]$ on $X_{N}^{S S}\left[\omega_{X}\right]$.

Theorem 5.6. In the setup of Proposition 5.4, there exists a Kähler structure ${ }^{9} \omega_{\bar{Q}}$ on the compact complex space $\bar{Q}$ whose restriction $\omega_{Q}=\left.\omega_{\bar{Q}}\right|_{Q}$ to $Q \hookrightarrow \bar{Q}$ is smooth and fulfils

$$
\left[\pi^{*} \omega_{Q}\right]=\left[\left.\omega_{X}\right|_{N} ^{s s}\left[\omega_{X}\right]\right] \in H^{2}\left(X_{N}^{s s}\left[\omega_{X}\right], \mathbb{R}\right) .
$$

Proof. Once again, recall our setup in the following commutative diagram

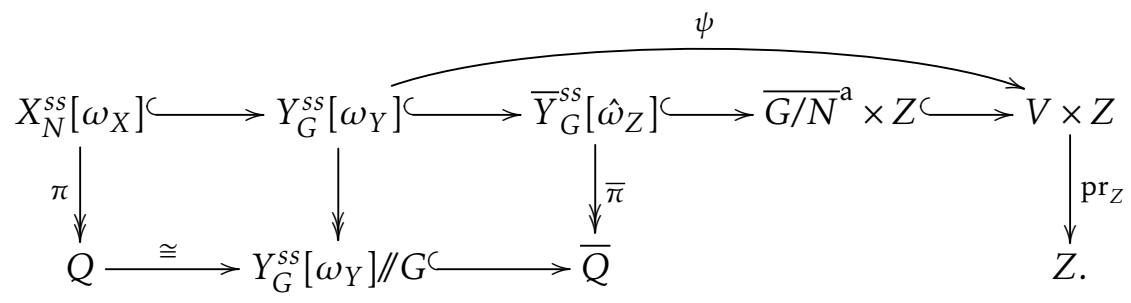

By applying the Kählerian reduction procedure of [HHL94] to $\bar{Y}_{G}^{s S}\left[\hat{\omega}_{Z}\right]$ and to the quotient $\bar{Q}$, we obtain a Kählerian structure $\omega_{\bar{Q}}$ on $\bar{Q}$ induced by restricting local $K$-invariant potentials of $\hat{\omega}_{Z}$ to $\mu_{\bar{Y}}^{-1}(0)$ and by the homeomorphism $\mu_{\bar{Y}}^{-1}(0) / K \simeq \bar{Q}$, cf. Theorem 2.6. We denote the restriction of $\omega_{\bar{Q}}$ to $Q$ by $\omega_{Q}$.

$9 \uparrow$ See [Gre10a, Sections 3.1 and 3.2] for the basic definitions regarding Kähler structures on (singular) complex spaces. 
In order to show that $\omega_{Q}$ is smooth, we first note that $Y_{G}^{s S}\left[\omega_{Y}\right] \subset \bar{Y}_{G}^{s S}\left[\hat{\omega}_{Z}\right]$ is smooth and $\bar{\pi}$-saturated, and secondly recall the observation made in the proof of Theorem 5.3 above that the G-action on $Y_{G}^{s S}\left[\omega_{Y}\right]$ is free. Therefore, it follows from the construction of the reduced Kähler form $\omega_{\bar{Q}}$, see [HHL94, Lemma 2 on page 132 and the proof on pages 133/134] and also compare with [Sj95, Theorem 2.10], that in the fundamental commutative diagram

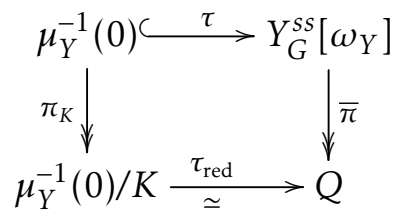

the fibre $\mu_{Y}^{-1}(0)$ is smooth, the $K$-action on $\mu_{Y}^{-1}(0)$ is free, and that the Kähler structure $\omega_{Q}$ is smooth and fulfils the "symplectic reduction" equation

$$
\tau^{*}\left(\bar{\pi}^{*} \omega_{Q}\right)=\pi_{K}^{*}\left(\tau_{\text {red }}^{*} \omega_{Q}\right)=\left.\omega_{Y}\right|_{\mu_{Y}^{-1}(0)}=\tau^{*}\left(\left.\omega_{Y}\right|_{Y_{G}^{s s}\left[\omega_{Y}\right]}\right) .
$$

More is true. Since $Y_{G}^{s s}\left[\omega_{Y}\right]$ is $\bar{\pi}$-saturated, and since the moment map $\mu_{\bar{Y}}: \bar{Y} \rightarrow \mathfrak{k}^{*}$ is proper as observed in the proof of Proposition 5.4, the moment map $\mu_{Y}$ is admissible in the sense that the gradient flow $F_{t}$ of $-\left\|\mu_{Y}\right\|^{2}$ through any point $p \in Y_{G}^{s s}\left[\omega_{Y}\right]$ exists for all times, cf. [Kir84, §9], and hence there exists a continuous retraction of $Y_{G}^{s s}\left[\omega_{Y}\right]$ to $\mu_{Y}^{-1}(0)$ defined by $z \mapsto \lim _{t \rightarrow \infty} F_{t}(z)$, see [Sj95, page 109] and the references given there, as well as [Ler05]. In particular, the inclusion displayed in the first line of Diagram (5.2) induces an isomorphism between de Rham cohomology groups,

$$
\tau^{*}: H^{2}\left(Y_{G}^{s s}\left[\omega_{Y}\right], \mathbb{R}\right) \stackrel{\cong}{\longrightarrow} H^{2}\left(\mu_{Y}^{-1}(0), \mathbb{R}\right) .
$$

Equation (5.3) therefore implies that

$$
\left[\bar{\pi}^{*} \omega_{Q}\right]=\left[\left.\omega_{Y}\right|_{Y_{G}^{s s}}\left[\omega_{Y}\right]\right] \in H^{2}\left(Y_{G}^{s s}\left[\omega_{Y}\right], \mathbb{R}\right) .
$$

In addition, from the right hand part of Diagram (5.1), from Equations (4.2) and (4.3), and from the the fact that the de Rham cohomology class of $\omega_{V}$ is trivial we infer that

$$
\left[\left.\omega_{Y}\right|_{Y_{G}^{s s}\left[\omega_{Y}\right]}\right]=\left[\psi^{*}\left(\operatorname{pr}_{Z}^{*}\left(\omega_{Z}\right)\right)\right] \in H^{2}\left(Y_{G}^{s s}\left[\omega_{Y}\right], \mathbb{R}\right),
$$

so that (5.4) becomes

$$
\left[\bar{\pi}^{*} \omega_{Q}\right]=\left[\psi^{*}\left(\operatorname{pr}_{Z}^{*}\left(\omega_{Z}\right)\right)\right] \in H^{2}\left(Y_{G}^{s s}\left[\omega_{Y}\right], \mathbb{R}\right) .
$$

Finally, using this, the left hand part of Diagram (5.1), and the fact that $Z$ as a $G$-extension of $X$ fulfils (3.1) we conclude

$$
\left[\pi^{*} \omega_{Q}\right]=\left[\left.\omega_{X}\right|_{X_{N}^{s s}\left[\omega_{X}\right]}\right] \in H^{2}\left(X_{N}^{s s}\left[\omega_{X}\right], \mathbb{R}\right) .
$$

\section{References}

[Akh95] D. N. Akhiezer, Lie group actions in complex analysis, Aspects of Mathematics, vol. 27, Friedr. Vieweg \& Sohn, Braunschweig/Wiesbaden, 1995. MR-1334091

[BBS85] A. Białynicki-Birula and A. J. Sommese, Quotients by $\mathbf{C}^{*} \times \mathbf{C}^{*}$ actions, Trans. Amer. Math. Soc. 289 (1985), no. 2, 519-543. MR-0784002

[BV89] D. Barlet and J. Varouchas, Fonctions holomorphes sur l'espace des cycles, Bull. Soc. Math. France 117 (1989), no. 3, 327-341. MR-1020110

[Bla56] A. Blanchard, Sur les variétés analytiques complexes, Ann. Sci. Ecole Norm. Sup. (3) 73 (1956), 157-202. MR-0087184 
[Bo91] A. Borel, Linear algebraic groups, second edition, Graduate Texts in Mathematics, vol. 126, Springer-Verlag, New York, 1991. MR-1102012

[BT82] R. Bott and L. W. Tu, Differential forms in algebraic topology, Graduate Texts in Mathematics, vol. 82, Springer-Verlag, New York, 1982. MR-0658304

[Br14] M. Brion, On automorphisms and endomorphisms of projective varieties. In: Automorphisms in birational and affine geometry, pp. 59-81, Springer Proc. Math. Stat., vol. 79, Springer, Cham, 2014. MR-3229345

[CD16] G. Codogni and R. Dervan, Non-reductive automorphism groups, the Loewy filtration and K-stability, Ann. Inst. Fourier (Grenoble) 66 (2016), no. 5, 1895-1921. MR-3533272

[Dem12] J.-P. Demailly, Complex analytic and differential geometry, 2012. Available online at http://wwwfourier.ujf-grenoble.fr/ demailly/manuscripts/agbook.pdf.

[DK07] B. Doran and F. Kirwan, Towards non-reductive Geometric Invariant Theory, Pure Appl. Math. Q. 3 (2007), no. 1, part 3, 61-105. MR-2330155

[Fuj78] A. Fujiki, On automorphism groups of compact Kähler manifolds, Invent. Math. 44 (1978), no. 3, 225-258. MR-0481142

[Fuj96] - Kähler quotient and equivariant cohomology. In: Moduli of vector bundles (Sanda, 1994; Kyoto, 1994), pp. 39-53, Lecture Notes in Pure and Appl. Math., vol. 179, Dekker, New York, 1996. MR-1397979

[FL81] W. Fulton and R. Lazarsfeld, Connectivity and its applications in algebraic geometry. In: Algebraic geometry (Chicago, Ill., 1980), pp. 26-92, Lecture Notes in Mathematics, vol. 862, Springer, Berlin-New York, 1981. MR-0644817

[Gre10a] D. Greb, Projectivity of analytic Hilbert and Kähler quotients, Trans. Amer. Math. Soc. 362 (2010), no. 6, 3243-3271. MR-2592955

[Grel0b]—, Compact Kähler quotients of algebraic varieties and Geometric Invariant Theory, Adv. Math. 224 (2010), no. 2, 401-431. MR-2609010

[GMO11] B. Gilligan, C. Miebach, and K. Oeljeklaus, Homogeneous Kähler and Hamiltonian manifolds, Math. Ann. 349 (2011), no. 4, 889-901. MR-2777037

[Gr83] F. D. Grosshans, The invariants of unipotent radicals of parabolic subgroups, Invent. Math. 73 (1983), no. 1, 1-9. MR-0707345

[Gr97] , Algebraic homogeneous spaces and invariant theory, Lecture Notes in Mathematics, vol. 1673, Springer-Verlag, Berlin, 1997. MR-1489234

[GS82] V. Guillemin and S. Sternberg, Geometric quantization and multiplicities of group representations, Invent. Math. 67 (1982), no. 3, 515-538. MR-0664118

[GS84] _ Symplectic techniques in physics, Cambridge University Press, Cambridge, 1984. MR0770935

[GJS02] V. Guillemin, L. Jeffrey, and R. Sjamaar, Symplectic implosion, Transform. Groups 7 (2002), no. 2, 155-184. MR-1903116

[HaH99] J. Hausen and P. Heinzner, Actions of compact groups on coherent sheaves, Transform. Groups 4 (1999), no. 1, 25-34. MR-1669182 
[Hei91] P. Heinzner, Geometric invariant theory on Stein spaces, Math. Ann. 289 (1991), no. 4, 631-662. MR-1103041

[HH96] P. Heinzner and A. T. Huckleberry, Kählerian potentials and convexity properties of the moment map, Invent. Math. 126 (1996), no. 1, 65-84. MR-1408556

[HH99] , Analytic Hilbert quotients. In: Several complex variables (Berkeley, CA, 1995-1996), pp. 309-349, Math. Sci. Res. Inst. Publ., vol. 37, Cambridge Univ. Press, Cambridge, 1999, pp. 309349. MR-1748608

[HHL94] P. Heinzner, A. T. Huckleberry, and F. Loose, Kählerian extensions of the symplectic reduction, J. Reine Angew. Math. 455 (1994), 123-140. MR-1293876

[HL94] P. Heinzner and F. Loose, Reduction of complex Hamiltonian G-spaces, Geom. Funct. Anal. 4 (1994), no. 3, 288-297. MR-1274117

[Hu05] Y. Hu, Topological aspects of Chow quotients, J. Differential Geom. 69 (2005), no. 3, 399-440. MR2170276

[HW90] A. T. Huckleberry and T. Wurzbacher, Multiplicity-free complex manifolds, Math. Ann. 286 (1990), no. 1-3, 261-280. MR-1032934

[Hum75] J. E. Humphreys, Linear Algebraic Groups, Graduate Texts in Mathematics, vol. 21, Springer-Verlag, New York, 1975. MR-0396773

[Jac62] N. Jacobson, Lie algebras, Interscience Tracts in Pure and Applied Mathematics, vol. 10, Interscience Publishers, New York-London, 1962. MR-0143793

[Kir84] F. C. Kirwan, Cohomology of quotients in symplectic and algebraic geometry, Mathematical Notes, vol. 31, Princeton University Press, Princeton, NJ, 1984. MR-0766741

[Kir11] _ Symplectic implosion and nonreductive quotients. In: Geometric aspects of analysis and mechanics, pp. 213-256, Progr. Math, vol. 292, Birkhäuser/Springer, New York, 2011. MR-2809474

[Ler05] E. Lerman, Gradient flow of the norm squared of a moment map, Enseign. Math. (2) 51 (2005), no. 1-2, 117-127. MR-2154623

[Lie78] D. I. Lieberman, Compactness of the Chow scheme: applications to automorphisms and deformations of Kähler manifolds. In: Fonctions de plusieurs variables complexes, III (Sém. François Norguet, 1975-1977), pp. 140-186, Lecture Notes in Math., vol. 670, Springer, Berlin, 1978. MR-0521918

[Mat60] Y. Matsushima, Espaces homogènes de Stein des groupes de Lie complexes, Nagoya Math. J. 16 (1960), 205-218. MR-0109854

[MFK94] D. Mumford, J. Fogarty, F. C. Kirwan, Geometric Invariant Theory, 3rd Edition, Ergebnisse der Mathematik und ihrer Grenzgebiete (2), vol. 34, Springer-Verlag, Berlin, 1994. MR-1304906

[OV90] A. Onishchik and È. B. Vinberg, Lie groups and algebraic groups, Springer Series in Soviet Mathematics, Springer-Verlag, Berlin, 1990. MR-1064110

[PV72] V. L. Popov and È. B. Vinberg, On a class of quasihomogeneous affine varieties, Izv. Akad. Nauk SSSR Ser. Mat. 36 (1972), 749-764; English translation in Math. USSR Izv. 6 (1972), 743-758. MR-0313260

[Sj95] R. Sjamaar, Holomorphic slices, symplectic reduction and multiplicities of representations, Ann. of Math. (2) 141 (1995), no. 1, 87-129. MR-1314032 
[Sj98] _ C Convexity properties of the moment mapping re-examined, Adv. Math. 138 (1998), no. 1, 46-91. MR-1645052

[Som75] A. J. Sommese, Extension theorems for reductive group actions on compact Kaehler manifolds, Math. Ann. 218 (1975), no. 2, 107-116. MR-0393561

[Sze14] G. Székelyhidi, An introduction to extremal Kähler metrics, Graduate Studies in Mathematics, vol. 152, American Mathematical Society, Providence, RI, 2014. MR-3186384

[Var84] J. Varouchas, Stabilité de la classe des variétés kählériennes par certains morphismes propres, Invent. Math. 77 (1984), no. 1, 117-127. MR-0751134

[Voi07] C. Voisin, Hodge theory and complex algebraic geometry II, Cambridge Studies in Advanced Mathematics, vol. 77, Cambridge University Press, Cambridge, 2007. MR-2449178

[Whi72] H. Whitney, Complex analytic varieties, Addison-Wesley Publishing Co., Reading, Mass.-LondonDon Mills, Ont., 1972. MR-0387634 